\title{
Theory of Constraints for Publicly Funded Health Systems
}

by

Somayeh Sadat

A thesis submitted in conformity with the requirements

for the degree of Doctor of Philosophy

Department of Mechanical and Industrial Engineering

University of Toronto

(C) Copyright by Somayeh Sadat 2009 


\title{
Theory of Constraints for Publicly Funded Health Systems
}

\author{
Somayeh Sadat \\ Doctor of Philosophy \\ Mechanical and Industrial Engineering \\ University of Toronto
}

2009

\begin{abstract}
This thesis aims to fill the gaps in the literature of the theory of constraints (TOC) in publicly funded health systems. While TOC seems to be a natural fit for this resource-constrained environment, there are still no reported application of TOC's drum-buffer-rope tool and inadequate customizations with regards to defining system-wide goal and performance measures.

The "Drum-Buffer-Rope for an Outpatient Cancer Facility" chapter is a real world case study exploring the usefulness of TOC’s drum-buffer-rope scheduling technique in a publicly funded outpatient cancer facility. With the use of a discrete event simulation model populated with historical data, the drum-buffer-rope scheduling policy is compared against "high constraint utilization" and "low wait time" scenarios. Drum-buffer-rope proved to be an effective mechanism in balancing the inherent tradeoff between the two performance measures of instances of delayed treatment and average patient wait time. To find the appropriate level of compromise in one performance measure in favor of the other, the linkage of these measures to system-wide performance measures are proposed.
\end{abstract}

In the "Theory of Constraints' Performance Measures for Publicly Funded Health Systems” chapter, a system dynamics representation of the classical TOC's system-wide goal and performance measures for publicly traded for-profit companies is developed, which forms the 
basis for developing a similar model for publicly funded health systems. The model is then expanded to include some of the factors that affect system performance, providing a framework to apply TOC’s process of ongoing improvement in publicly funded health systems.

The "Connecting Low-Level Performance Measures to the Goal" chapter attempts to provide a framework to link the low-level performance measures with system-wide performance measures. It is claimed that until such a linkage is adequately established, TOC has not been fully transferred to publicly funded health systems. 


\section{Acknowledgments}

I am strongly grateful to my extremely supportive and knowledgeable supervisor, Professor Michael Carter, for providing the best environment for me to learn in and conduct research. With his support, I had the freedom to get exposed to a variety of research ideas related to health services that went far beyond the core industrial engineering areas, including health economics, health policy making, and strategic issues in health care management. Indeed, all of these subjects proved to be an essential part of connecting the dots in the fourth chapter of this thesis. I would like to thank him for encouraging me to learn anything that could help better understand the complex health care environment. The health care industry is indeed too complex to be defined with the boundaries of one subject area.

I am very thankful to my committee members, Professor Brian Golden and Professor Daniel Frances, for their advice throughout the course of the research. The constructive feedback I got in my various doctoral committee meetings greatly helped me to define the scope of the research. Professor Golden has also been very instrumental to support the logistics of conducting this research. Without his efforts, I might not have had the chance to work on a real world case study. The frequent chats I had with Professor Frances helped me better compare various process improvement techniques and define the topic of my thesis. I like to thank him for caring to share his insights with me.

I would also like to thank the external examiner of this thesis, Professor Sally Brailsford who came all the way from the University of Southampton in UK to participate in the defense session. Her detailed feedback proved very useful in preparing the final version of this thesis. I also like to extend my gratitude to Professor Baris Balcioglu for serving on my final examination committee. 
The third chapter of this doctoral research is part of an umbrella patient centered care research project that was generously funded by MDS Labs to be conducted by Rotman School of Management at the University of Toronto. I would like to thank the research team working on the first phase of the umbrella research project, specifically Professor Golden (Principal Investigator), Rosemary Hannam (the research coordinator), and Professor Dilip Soman from Rotman School of Management, Dr. Mark Minden from Princess Margaret Hospital, and Dr. Tom McGowan from Credit Valley Hospital. I learnt a lot about the context of the problem I was analyzing by working along each of them. Rosemary Hannam was especially helpful in getting me in contact with appropriate hospital staff to obtain the type of data I was looking for. I would like to express my gratitude for all her efforts in smoothing the process of conducting this research as much as possible, especially for taking care of the various ethics approval applications. I would also like to thank Mansoor Ahmed from the department of mechanical and industrial engineering at the University of Toronto for helping out with the initial data analysis.

Most important, the development of the simulation model was not possible without the support of the management and various staff at Princess Margaret Hospital, including (listed in alphabetical order): Sally Balmer, Karen Chuk, Darlene Dale, Sara Downey, Dawn Gilmou, Penny Hackenbrook-Rogers, Sharon Howe, Roy Lee, Andrew Macdonald, Jayesh Patel, Paul Radulescu, Carolyn Sibbick, Jan Stewart, Gisele Thorpe, Sheila Weinstock. I wish to thank them all.

I am thankful to my friends for their emotional support throughout the course of my graduate studies. Special thanks go to Hossein, for pushing me to get the job done on a daily basis, and Golnaz, for keeping my spirit up when I had reasons to feel otherwise. I am also thankful to Ali, with whom I used to discuss my thesis over tea and who once had a good idea about one of the 
details of my model. Special thanks also go to Hazhir, who helped me with justifying my methodology at a critical point of this process. I also feel indebted to Afsaneh, who shared her insights about the $\mathrm{PhD}$ experience with me; letting me see the rational of it all from day one. I am also grateful to my good friends Sara, Iman, Parisa, and Maryam.

Last but not least, it goes without saying that I am forever indebted to my wonderful family, especially my parents. The confidence and calmness they harvested in my character during my upbringing was the personal trait needed to complete a PhD. Thank you! 
To my family 


\section{Table of Contents}

\section{Contents}

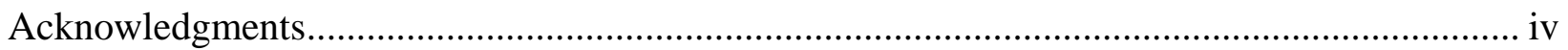

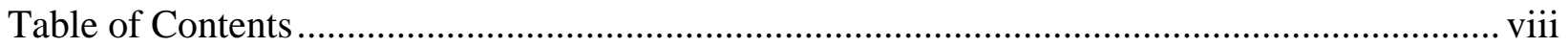

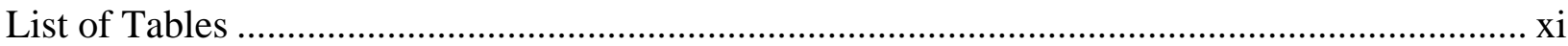

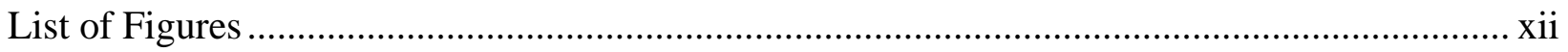

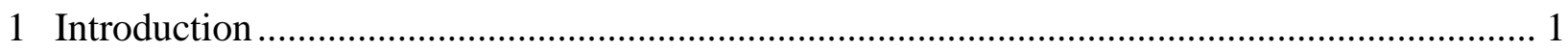

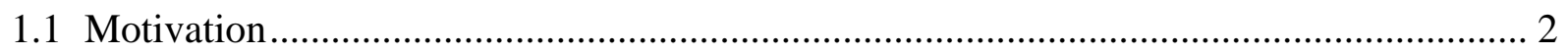

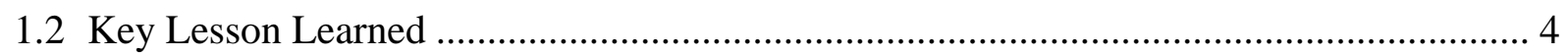

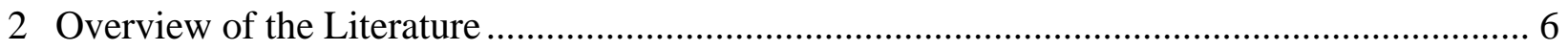

2.1 Historical View of the TOC Literature ………………....................................................... 7

2.1.1 Process of Ongoing Improvement ..................................................................... 7

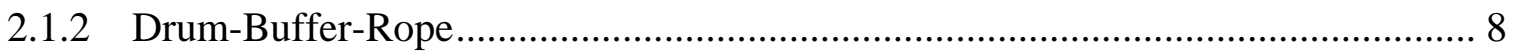

2.1.3 Goal and Performance Measures ........................................................................... 9

2.1.4 Thinking Process.............................................................................................. 10

2.1.5 Critical Chain ................................................................................................. 12

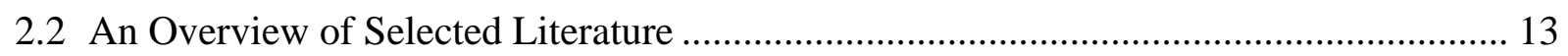

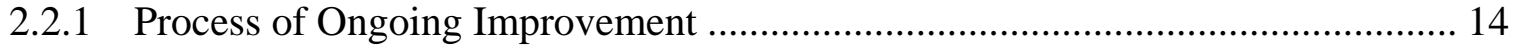

2.2.2 Drum-Buffer-Rope........................................................................................... 17

2.2.3 Goal and Performance Measures .......................................................................... 17

2.2.4 Thinking Process................................................................................................ 23

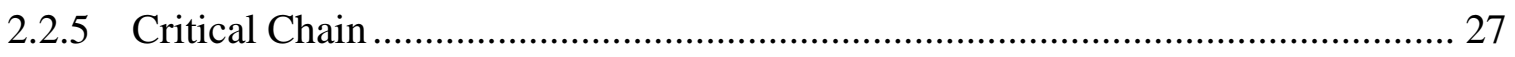


2.3 TOC for Publicly Funded Health Systems: Literature Gaps ....................................... 28

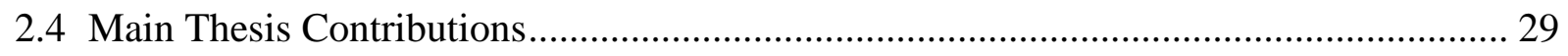

2.4.1 Chapter 3: Drum-Buffer-Rope................................................................ 29

2.4.2 Chapter 4: Goal and Performance Measures Development ............................... 30

3 Drum-Buffer-Rope for an Outpatient Cancer Facility ................................................. 31

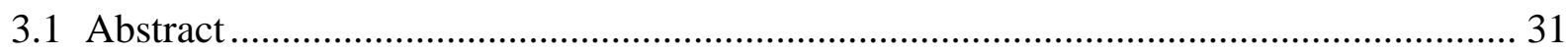

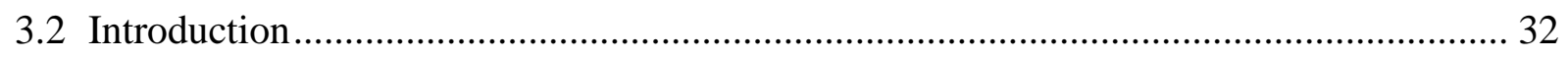

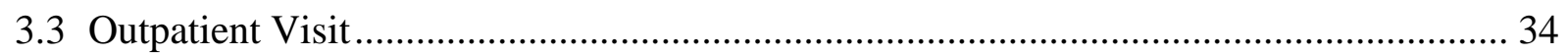

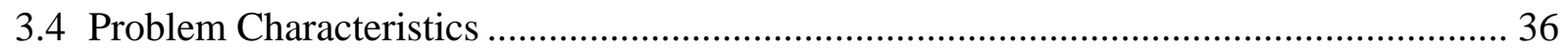

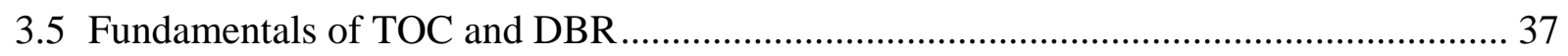

3.6 TOC in Health Care: A Review of Literature ............................................................ 40

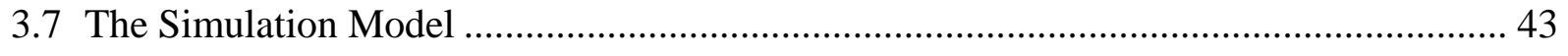

3.8 Data Needs, Sources, and Assumptions................................................................ 46

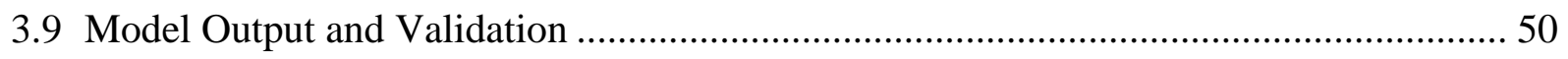

3.10Performance Measures and Scenario Definitions .................................................... 53

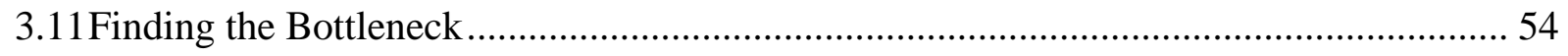

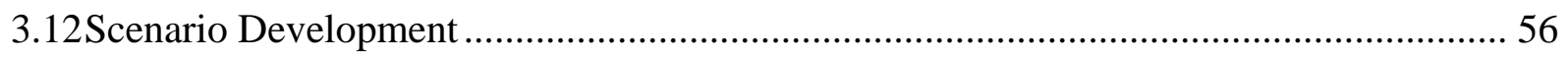

3.12.1 High Constraint Utilization Scenario ......................................................... 56

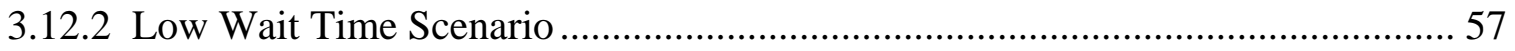

3.12.3 Drum-Buffer-Rope Scenario................................................................. 58

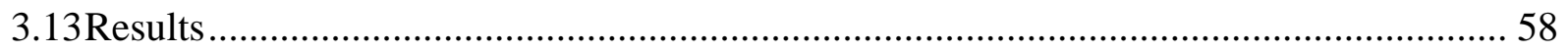

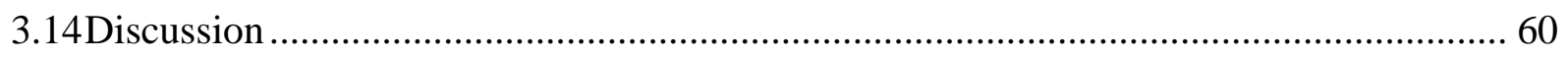

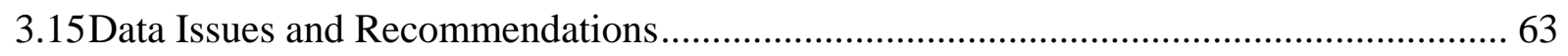

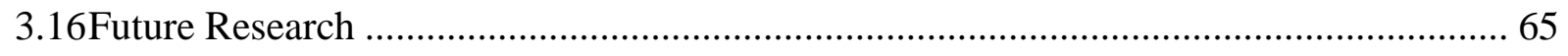

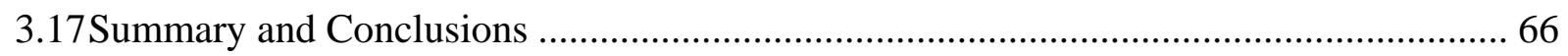

4 TOC’s Performance Measures for Publicly Funded Health Systems .................................... 67 


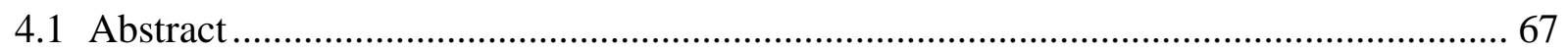

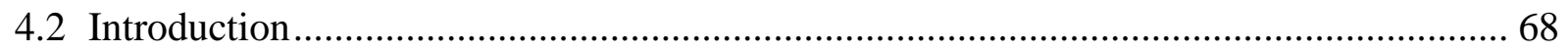

4.3 An SD Model of TOC’s Classical Goal and Performance Measures ................................ 69

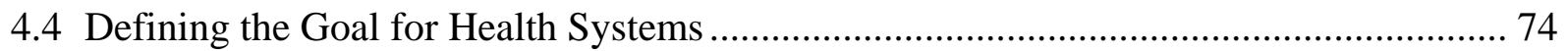

4.5 An SD Model of TOC’s Performance Measures for Health Systems ............................... 75

4.6 A Framework for Applying TOC’s Process of Ongoing Improvement in Health

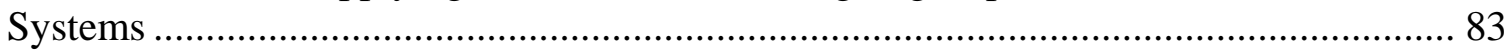

4.7 Discussion and Future Research ........................................................................................ 89

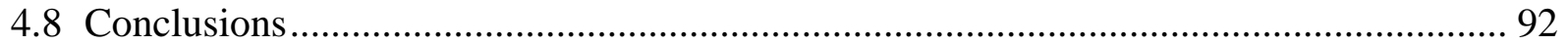

5 Connecting Low-Level Performance Measures to the Goal....................................................... 94

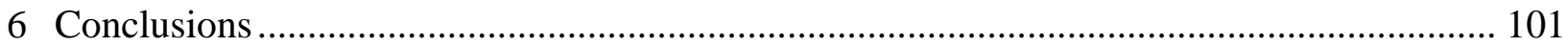

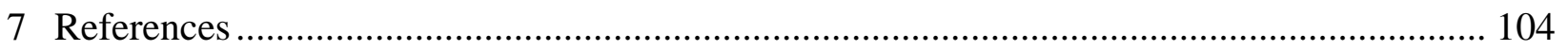




\section{List of Tables}

Table 3.1: Model Validation ................................................................................................ 52

Table 5.1: Total Hours Wasted to Receive Treatment at the TFU in Different Scenarios ........... 97 


\section{List of Figures}

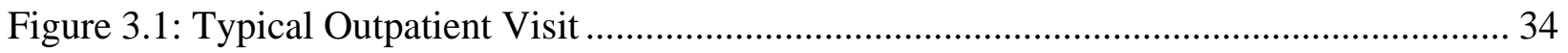

Figure 3.2: Simulated Performance Measures for the Three Scenarios.......................................... 59

Figure 3.3: Cumulative Distribution of Patient Wait Time ............................................................. 60

Figure 3.4: Performance Measure Values Resulting from Using Different Percentile Values of the Nth Treatment Chair Availability Times as Time Estimators in DBR ..................................... 61

Figure 4.1: TOC’s Classic Goal and Performance Measures ........................................................ 73

Figure 4.2: Health System’s Goal \& Performance Measures - Individual’s Perspective............. 76

Figure 4.3: TOC’s Goal and Performance Measures for Health Systems ..................................... 81

Figure 4.4: A conceptual model to guide TOC’s improvement process in health systems ........... 85

Figure 5.1: Low-Level \& System-Wide Performance Measures- Major Relationships................ 95

Figure 5.2: Low-Level \& System-Wide Performance Measures- Possible Relationships ............ 98 


\section{Introduction}

There are many advantages to publicly funding health systems, among those the fact that more people can receive health services at a considerable less cost. This is evident by comparing United State's health system with that of the other OECD countries. However, there are concerns about the sustainability of publicly funded health systems. Despite the relatively high proportion of the governmental budgets spent on health services, there is still a gap between demand and capacity, at the very least equal to the explicit demand put on hold on various wait lists. With an increased average life expectancy, part of which could be attributed to the success of the health system itself, comes an aging population that demands even more health services. Available information about latest drugs and technologies are raising expectations to have access to the best possible services, usually at significantly higher costs. Aging and close-to-retirement health human resources only make the prospects of keeping up with the rising demand more challenging, unless significant investments are made to enhance capacity. Accountability necessitates that the existing resources within the health system are best exploited before investing in additional capacity. As such, getting the most out of the available resources in publicly funded health systems is imperative.

Theory of constraints (TOC), also known as management by constraint or constraints management, is a relatively new management theory that emphasizes the importance of improving system performance through a smarter utilization of existing resources, especially by exploiting the bottleneck, before increasing system's capacity. The principal tenet of TOC is that any system has at least one constraint which limits its performance. Since a system can at best perform only as well as its constraints, TOC emphasizes that improving constraint's performance directly results in enhancing total system performance. 
The theory seems to be a natural fit for the resource-constrained publicly funded health systems and in fact some of its tools and concepts have been applied to this setting. However, there are still no reported application of TOC's drum-buffer-rope tool and inadequate customizations with regards to defining system-wide goal and performance measures. This doctoral thesis aims to fill these gaps in the literature so that the theory can be better exploited to enhance the performance of publicly funded health systems. To better clarify the contributions of the thesis, a brief overview of the published literature is provided in the chapter two, based on which applying the drum-buffer-rope technique as well as defining the TOC's system-wide goal and performance measures in the publicly funded health environments are identified as the current literature gaps. Chapter three, titled "Drum-Buffer-Rope for an Outpatient Cancer Facility", and chapter four, titled "Theory of Constraints' Performance Measures for Publicly Funded Health Systems", address the two mentioned literature gaps respectively. In chapter five we present a framework to assess the effects of applying a specific tool, such as the drum-buffer-rope technique, on the global goal. Chapter six presents the conclusions of this thesis.

The remaining of this chapter provides some background information on how the topic of this thesis was selected and the various phases of its development. We conclude the chapter with the key lesson learned during the course of this research.

\subsection{Motivation}

This thesis started as the quantitative leg of an umbrella patient centered care research project [Golden et. al., 2009] in a Canadian cancer hospital. The motivation for the patient centered research project came from a common perception that even though the quality of clinical care received by malignant blood disorder patients was excellent, there was room for improving the 
overall experience. Consequently, a multidisciplinary team of researchers with expertise in oncology, marketing, operations research, and change management came together to recommend strategies for a more patient centered model of care delivery. The research project was generously funded by MDS labs, to be conducted by Rotman School of Management at the University of Toronto. The research team included Professor Brian Golden (Principal Investigator), Professor Michael Carter, Dr. Tom McGowan, Rosemary Hannam (Research Coordinator), Dr. Mark Minden, and Professor Dilip Soman.

The patient centered research project was completed in two phases. In phase one, staff and patients were interviewed to understand patient expectations, patient experience, and the gap between the two. Besides a variety of qualitative issues brought up in the interviews, there were concerns about the amount of time that patients had to wait in order to receive treatment in an outpatient visit. The wait time was sometimes so long that patients were asked to leave the facility and return the next day to receive the treatment. Given the high frequency of these outpatient visits and the fact that waiting was another burden for the already fatigued malignant blood disorder patients, the second phase of the research project was defined to find strategies that would help the hospital best utilize its capacity to reduce average outpatient wait time and instances of outpatients having to leave the facility and return the next day for treatment. In consultation with the hospital management, the patient group under study was limited to acute leukemia outpatients.

We did not, however, intend to only solve the problem for this specific case, as this could be a problem faced by other health institutions as well. To be able to generate knowledge that could be transferred to other institutions, we aimed at finding a methodology that would help better plan the operations in settings like the one we observed at the hospital under study. We browsed 
the literature for possible improvement methodologies that have worked in other industries, and found the theory of constraints as one that would focus the improvement efforts on the heart of the problem. We then found one of the tools of the theory of constraints (the drum-buffer-rope technique) that would best tackle the problem at hand in the hospital under study, and for the first time in the published literature applied this tool to a health care institution. As such, we contributed to the TOC applications literature. But, more importantly, we demonstrated the method of applying this tool to tackle the problem at hand and the potential benefit of its application in planning the operations of the hospital under study, which should be applicable to other similar institutions. However, we found it difficult to assess the impacts of applying this tool on the overall health system performance, mainly due to the fact that the published literature has not adequately developed the equivalent TOC’s system-wide goal and performance measures for publicly funded health systems. As such, we embarked on addressing this gap in the literature, and made a theoretical contribution both to the theory of constraints literature and to the management and policy making in publicly funded health systems. Finally, we introduced a framework to link the low-level performance measures with system-wide goal and performance measures, thereby enabling us to assess the impacts of applying a specific tool, such as the drumbuffer-rope, on the system as a whole.

\subsection{Key Lesson Learned}

We draw one important lesson from this course of research. It is very important to understand the fundamental logic of any theory before fully customizing it to different settings. It is usually easy to take one tool or technique from a theory and apply it in a different setting, but this is of little 
value if the effects of this application cannot be gauged in the larger picture of the rationale of the theory. Theory of constraints, for instance, is about leveraging the constraint to improve the performance of the system. While each of its tools and concepts can be, and for the most part have been, applied to the setting of publicly funded health systems, the theory can only be said to have been customized to this setting when the purpose of the various tools can be gauged with regards to the purpose of the theory. In other words, one should be able to answer if applying a tool of TOC has in fact improved overall system performance through leveraging system's constraint. It is our belief that without a proper definition of the goal and performance measures in this setting, such a linkage of the tools to the rationale of the theory is not completely possible. The framework we provide in the fourth chapter is only a first step towards the purpose of fully formulating TOC's goal and performance measures for publicly funded health systems, and the fifth chapter is only an example of what questions should be asked to establish the linkage of the specific tools of the theory, such as the one applied in the third chapter, to the rationale of the theory. But they are definitely steps in the right direction. 


\section{Overview of the Literature}

This chapter provides a brief overview of the literature on the development and applications of the theory of constraints in order to identify the gaps in applying the theory to publicly funded health systems. It is by no means a comprehensive review of the literature. The volume of published literature on TOC is very large and on the increase in recent years. About 1800 of TOC-related papers are found on the Scopus as of November 2008, and these exclude the books on the topic.

At an aggregate level, TOC’s management tools can be categorized into three branches: TOC's process of ongoing improvement (the goal, performance measures, and the five focusing steps), logistics (drum-buffer-rope and buffer management techniques), and problem solving/thinking tools. Since the goal is to identify literature gaps, literature from a all TOC branches had to be investigated.

To best categorize and present the literature, a chronological view of the development of various TOC tools and concepts is presented in section 2.1, with reference to some of the seminal books that best introduce the tools and concepts. Subsequently, section 2.2 provides an overview of the literature on each TOC tool or concept. Building on the cited publications, the gaps in the literature with regards to customizing and applying the TOC to publicly funded health systems are identified and discussed under section 2.3. Section 2.4 introduces the two main chapters of this thesis, “Drum-Buffer-Rope for an Outpatient Cancer Facility” and “Theory of Constraints' Performance Measures for Publicly Funded Health Systems”, and summarizes their contribution in addressing the identified literature gaps. 
It should be noted that the relevant literature to each chapter of the thesis are also included in the actual chapter, so that the concepts in each chapter are well presented in the context of the published literature.

\subsection{Historical View of the TOC Literature}

TOC is a management theory that has evolved from the floor plan up to the strategic levels in recent years. While a lot of researchers have contributed to the development of the theory, we follow Watson et al's (2007) approach in summarizing the development of the theory with specific references to the books by Dr. Eliyahu Goldratt, as they provide milestones of the key contributions of the theory to the field of management. The subheadings for each subsection below summarize the tool or concept in one term, which help to later categorize the literature applying or contributing to each tool and concept under the same subheading in section 2.2.

We do not aim to fully explain any of the tools or concepts here, as they have all been very well discussed in the cited literature. We only mention the main idea of each tool or concept to build the vocabulary necessary to later explain the contribution of each of the selected literature in enough details, with the ultimate purpose of finding the literature gaps in applying TOC to publicly funded health systems. The details of the selected tool (drum-buffer-rope) and concept (goal and performance measure definition) that are the main subject of this thesis are described in more detail in chapters three and four, respectively.

\subsubsection{Process of Ongoing Improvement}

TOC started by a simple request for help to increase the output of a particular plant through better scheduling. Goldratt, a physicist by education, developed a program that increased the 
plant's output significantly in a short time. The program was later commercialized under the name of Optimized Production Technology and sold to interested companies. The program basically collected and arranged data in a specific format in order to first schedule the bottleneck locations, and then move backwards to schedule the proceeding non-bottleneck steps, all the way back to the point of material release. Despite the numerous success stories, there were incidences of failure that at least could partly be attributed to a lack of adherence to the schedule. There were good reasons for this lack of compliance though, as the rationale for the schedule was not well understood by the management and those implementing it. It seemed that the schedule kept some stations highly utilized, while others remained idle. The performance management systems in place, however, still gauged workers' performance with regards to local productivity measurements. This gave the workers at the non-busy stations a disincentive to stick to the schedule. Non-compliance brought failure, but on the positive side, urged Goldratt to educate the companies about the rationale behind the scheduling technique. The resulting business novel, “The Goal” (Goldratt and Cox, 1984), served more than merely educating the companies who had bought the scheduling software and became a bestseller by itself. Different companies started following its process of ongoing improvement comprising five steps: identifying the constraint, exploiting the physical constraint and eliminating the policy constraint, subordinating the system to the constraint, elevating the constraint, and overcoming inertia by starting over from the first step of the process. (Watson et al., 2007)

\subsubsection{Drum-Buffer-Rope}

The details of the drum-buffer-rope technique, TOC's scheduling methodology for physically constrained systems, were spelled out in another book: "The Race” (Goldratt and Fox, 1986). Drum, or the bottleneck, sets the pace of the system and is protected by the buffer. The rope is the communication mechanism to signal the release of raw material at the right time. 
Buffer management is a related TOC application that determines the size and monitors the status of buffers. Determining the appropriate level of buffers is an ongoing research area; for instance see Radovilsky (1998), Gunn and Nahavandi (2000), Louw and Page (2004), and Ye (2007).

In practice, buffer levels can be set to some initial levels, for instance according to the rules of thumb mentioned in Ricketts (2008). Over time, buffers can be monitored and their right level can be determined through experience. Additionally, by comparing actual versus planned buffer sizes at set times, management can identify problems before they become critical, and through early feedback to the problematic process(es) reduce the need to expedite. Improvement efforts can then be focused on the more problematic processes. Monitoring buffers also makes it possible to assess the impacts of improvement initiatives as they are being implemented. (Watson et al., 2007)

\subsubsection{Goal and Performance Measures}

Later, Goldratt explicitly discussed the two prerequisites for implementing the process of ongoing improvement, namely defining the goal and performance measures, in a new book: "The Haystack Syndrome” (Goldratt, 1990). The book emphasized the fact that the goal of any system should be determined by its owners. It claimed that shareholders, as the true owners of publicly traded for-profit companies, define this goal as making money, both now and in the future. Moreover, the book introduced a set of operational performance measures- namely throughput, inventory, and operating expense- that enabled management to assess the effects of local actions globally, through the global performance measures of cash flow, net profit, and return on investment.

Throughput was defined as the rate at which the system generates money through sales, or more specifically, the selling price minus total variable costs. Total variable cost is the money that was 
not generated by the system, such as purchased parts and raw materials. Inventory was defined as all the money invested in purchasing things the system intends to sell, or more specifically, investments such as machines, equipment, etc., and finished goods and work-in-process inventory. Operating expenses was defined as all the money the system spends in turning inventory into throughput, more specifically wages, salaries, utility expenses, depreciation, etc. (Gupta, 2003).

The global performance measure of cash flow was defined to address the survival factor: whether or not the company goes bankrupt. The other two global performance measures help compare companies against each other for investment purposes. Net profit was defined as throughput minus operating expenses. Return on investment was defined as the ratio of net profit over inventory.

\subsubsection{Thinking Process}

Recognizing that the constraint in most systems is policy or procedural (Motwani et al., 1996), the focus of the theory gradually shifted to develop more qualitative tools to tackle the policy constraints and resistance to change. Goldratt’s next book titled “It’s Not Luck” (Goldratt, 1994), strived to create a formalized thinking process to answer the three fundamental questions that management is faced when dealing with constraints:

1. What to change?

2. What to change to?

3. How to cause the change? 
Five main logic tools were developed: Current Reality Tree, Evaporative Cloud (also known as Conflict Resolution Diagram), Future Reality Tree, Prerequisite Tree, and Transition Tree. The tools are meant to facilitate the logical thinking required to answer the above questions.

The first question (i.e.: what to change?) is answered by describing the present situation with a current reality tree: a logical structure that depicts the state of reality as it currently exists in the system (Dettmer, 1997). It is basically a map of cause and effect relationships that explain how the core problem (root cause) is causing the existing undesirable effect(s) (UDEs) through some intermediate effects.

The second question (i.e. what to change to?) is answered by the use of evaporating cloud and future reality tree tools. Evaporating cloud addresses conflict situations by surfacing hidden assumptions. This leads to innovative solutions, known as injections, capable of evaporating the conflict. The future reality tree then tests the injections by identifying the consequences using the same cause and effect type of relationships used in the current reality tree.

The third question (how to cause the change?) is addressed by the prerequisite tree and transition tree tools. The prerequisite tree identifies obstacles in realizing an objective, remedies to obstacles, and a sequence of actions. It bridges the gap between the future reality tree and the transition tree, which is the step-by-step implementation plan to enable effective navigation through a change process.

In short, as Kim et al (2008) summarize it, the thinking process first identifies problematic symptoms, the evidence that the system is not performing as desired. Then various thinking process tools are used to "deduce the causes of those symptoms, what needs to be done to correct 
those causes, and how such corrective actions could be implemented”, by following strict logic rules and the rigour of cause-and-effect thinking.

\subsubsection{Critical Chain}

Finally, Goldratt’s “Critical Chain” book (Goldratt, 1997) introduced the contribution of the theory to the field of project management. The fundamental idea is that the emphasis should be on completing the project on time, not individual activities. As such, most of the excess time allocated to each activity in the traditional critical path method of project management is taken away to create a buffer for the whole project, usually resulting in a reduction in the overall time. The success of cutting the overall safety time is dependent on a number of behavorial factors, such as the level of confidence that concerned parties have in this process (Rand, 2000).

The project buffer is monitored throughout the project. The buffer is usually divided to three zones: safety, tracking, and expedite zone. A project that has not been completed yet is referred to as a hole. Holes in the safety zone are considered natural. When a hole reaches the tracking zone, it is investigated. But no action is taken until the hole reaches the expedite zone, meaning that the delays have depleted the project buffer to the extent that project completion time is jeopardized. At this point, management actions such as reassigning resources are implemented. This comes as no surprise to management since the delays have already been noticed and investigated when the hole was in the tracking zone. The feedback mechanism present in buffer management in non-project environments, where improvement efforts are focused on problematic processes for the sake of improving future performance, is generally absent in the project environment because by their definition, projects are one-time unique sets of activities. (Umble and Umble, 2006) 


\subsection{An Overview of Selected Literature}

TOC has been successfully applied in a variety of settings. Mabin and Balderstone (2003) looked into the published literature to find successful and failed TOC applications. They were unable to find any reports of failure. They presented the results of a meta-analysis of over 80 successful TOC applications, based on available quantitative data, which demonstrated that applying TOC has resulted in significant improvements in both operational and financial performance.

Lubitsh et al (2005) investigated the impact that applying TOC has had on three NHS Trust departments, Neurosurgery, Eyes, and ENT, with regards to reducing waiting lists in the system and improving throughput of patients. Using data that was collected on a number of NHS performance indicators before and after the intervention (over a period of 40 months), they found that for both Eyes and ENT, most measures improved. However, significant improvements could not be verified for Neurosurgery, possibly due to the size of the system, complexity of treating neurological disorder, heavy reliance on support services, impact of emergencies on elective work, and the motivation and receptiveness of staff to the proposed changes. This finding emphasizes the importance of taking into account the social environment in order to maximize the benefits of TOC in an organization.

The impact of environmental factors on the degree of success of TOC applications has also been studied by Kalina-Kaminsky (2005) in an aggregate data analysis of TOC implementations. To explore the effect of external environment and culture combined with internal corporate culture on success, in terms of financial and operational performance, forty-eight extensive surveys were conducted. It was found that "positive financial and operational results are related to a nurturing environment supported by improved communication, coordination, cooperation, and 
participatory management style. In other words, if the organization takes care of its people, then its people take care of the organization in terms of improved market share, bottom line improvements, and financial stability.”

This section provides an overview of the selected publications on TOC, categorized under their main area of contribution. Some papers apply more than one tool or concept, but for the most part there is one major tool or concept that they can be identified with and that is the category they have been assigned to. In keeping with our purpose of finding the gaps in the literature, we only provide summaries of the papers that either apply TOC in the health industry, or can help to customize TOC to health systems (e.g. those discussing required TOC customizations for service or non-profit environments). The other papers are only cited (and not summarized) to demonstrate the wide applications of TOC to different settings. In selecting these latter papers from a huge number of TOC publications, preference was given to more recent ones, as Mabin and Balderstone (2000) have provided a comprehensive review of the older ones.

\subsubsection{Process of Ongoing Improvement}

Applications of the process of ongoing improvement have been numerous. Most have been the context of applying one or more of the other TOC tools (drum-buffer-rope, critical chain, or the thinking process) and as such have been categorized under the specific category of the corresponding TOC tool. Among the more general ones applying TOC’s process of ongoing improvement, we like to mention the two very recent one, one reporting on a TOC based expert system for an oil refinery (Rosolio et al, 2008), and the second discussing the improvement of the effectiveness of a loan and application process in a banking organization (Reid, 2007). We found four papers that demonstrated the full or partial application of TOC’s process of ongoing 
improvement in the health industry: Womack and Flowers (1999), Rotstein et al (2002), Kershaw (2000), and Gupta and Kline (2008).

Womack and Flowers (1999) present a case study in applying the TOC in a capitated managed care environment. Realizing the importance of ease of getting appointments as the number one customer satisfaction determinant, $366^{\text {th }}$ MDG tackled the issue by applying the TOC in two of its processes: scheduling process and patient-provider encounter process. Consequently, the appointment time was reduced from an average of 20 minutes to 15 minutes, while providers reported no reduction in actual time with the patient. Therefore, without compromising patient satisfaction, there was capacity to enroll more patients and increase the revenue with minimal cost (an additional $\$ 1.6$ million for a cost of less than $\$ 200,000$ ).

Kershaw (2000) applied the focusing steps to an outpatient oncology clinic administering chemotherapy to patients. The process is composed of six steps: checking in with the receptionist, blood tests in the lab, pretreatment process conducted by nurses in the exam room, physician consult in the exam room, chemotherapy administration in the treatment room, and scheduling a follow-up appointment with the receptionist. To identify the bottleneck, patient volumes were compared to the capacity of resources at each step of the treatment process and the treatment chairs were found to be the primary constrained resource. Improvement efforts were focused on the constraint. First, the three components of the treatment time- establishing intravenous access, administering prescribed drugs, and performing post treatment educationwere analyzed. It was decided that the post treatment education could be performed during drug administration and that the intravenous access, for more routine patients, could be established in the blood lab by lab technicians. Moreover, mobile supply caddies were set up so that time was 
not lost due to nurses looking for equipment and supplies when patients experienced undesirable side effects. At the end of the study, the capacity was increased by 20 to 25 percent.

Rotstein et al (2002) present a retrospective study of whether or not the bottleneck in a particular emergency department had been the physicians, to evaluate under what conditions adding medical staff would be a valuable strategy. This is a contribution, though probably not very generalizable, to the first step of the process of ongoing improvement: identifying the bottleneck. The marginal benefit of shortening non-trauma patients' length of stay during a period when a physician in the evening shift had been added was examined for different patient volumes using the SAS software package. To exclude other parameters that might have contributed to this change in the length of stay, the length of stay of the patients visiting in the morning shift served as a control group, as no staff were added to the morning shift during the period that the staffing level in the evening shift was examined. The study showed that the physician could only be regarded as the bottleneck within the 80-119 patient volume range.

Gupta and Kline (2008) present the application of TOC's process of ongoing improvement to a Chemical Dependency unit within a community mental health center. The paper makes recommendations in line with the five focusing steps of TOC's process of ongoing improvement. In identifying the constraint, wait times are taken as a clue to spot psychiatrists as the primary constraint of the system, and the therapists as the secondary. In the later steps of subordinating the system to the constraints, it is revealed that the clinical support function also demonstrates inadequate capacity.

A number of recommendations are made, including moving the psychiatrist's office closer to the waiting area which proved to save enough time for the psychiatrist to personally schedule new patients for Psychiatric Evaluation and make reminder calls for these patients. As a result, the 
no-show rate for Psychiatric Evaluation appointment fell from \%43 to \%20. Other recommendations regarding increasing pay of clinical staff support await agency action at the executive level. Also, reminder postcards were recommended for the unreachable patients. But more fundamentally, it was advised that the patients with a certain number of missed appointments per year should have their cases closed.

\subsubsection{Drum-Buffer-Rope}

Most recent reported applications of the durm-buffer-rope technique are in manufacturing settings such as a job shop environment (Chakravorty, 2001), hi-tech industry (Pass and Ronen, 2003), and a batch/mix process company (Belvedere and Grando, 2005). We could not find a reported application of the drum-buffer-rope technique in the health industry. There have been, however, suggestions of applying the drum-buffer-rope technique to the health industry. Siha (1999) summarizes the complexities encountered in the medical environment, namely stochastic cure times, dependent events, and probabilistic care plans, and suggests that the drum-bufferrope technique might be useful in this environment.

\subsubsection{Goal and Performance Measures}

The goal of health systems has not been widely discussed in the TOC literature. Some of the few suggested goals include "maximize life expectancy and quality of life at an acceptable cost to society” (Hunink, 2001), "to maximize the units of health” (Breen et. al., 2002), "providing safe care while thinking of money only as an operating expense - something to be reduced” (Mcnutt et. al., 2004), "to treat more patients both now and in the future” (Wright and King, 2006), to “maximize quality medical services provided to its customers, subject to budgetary constraints” (Ronen et. al., 2006), and “to make more health, today as well as in the future” (Schaefers et. al., 2007). 
The definitions of some of the operational performance measures, especially the inventory, get complicated when dealing with service industries, even the for-profit ones. Siha (1999) presented a classified model to apply TOC to Schmenner's (1986) classification of service organizations, in which she defined the performance measures for one of the categories. Schmenner (1986) uses two key elements to classify service delivery processes: labor intensity, and customer interaction and customization. The resulting two-by-two matrix is composed of four types of service organizations: service factory, service shop, mass service, and professional service.

Schmenner's (1986) service factory category is characterized by low degree of labor intensity and low degree of interaction and customization. Examples include airlines, trucking, hotels, and resorts and recreation. Siha (1999) defines the inventory for these organizations as the unused "service", such as a seat on a flight or space in a truck, and a room in a hotel or resort. The throughput is the money generated from selling the "service". And the operating expense has the standard TOC definition.

Sale (2005) tries to define the material and goods inventory in various service organizations, as the investments in equipment and most other items that are identified by TOC as inventory is common to both manufacturing and service organizations. Three possible flows within service organizations are identified: flow of material, flow of people, and flow of information.

For the flow of material type of services, post service is examined. An analogy is made between letters that need to be delivered and are waiting to enter the system and the materials inventory in manufacturing and another analogy between the letters already in the process of delivery and the work-in-progress inventory in manufacturing. Finished goods inventory is claimed to be nonexistent, as the completion of the process coincides with the delivery of the letter. 
Examples of processes that involve a flow of people include hospitals, banks, and airlines. Similar to the material flow case, the inventory is defined as people waiting to enter the first stage of the process, and people who have already been involved in some stage(s) of the process but have not still completed the process, analogous to the materials and work-in-process inventory in manufacturing respectively.

Finally, examples of flow of information are consultants, engineers, doctors, lawyers, architects, etc., for whom their knowledge is their service. Taking the instance of architects, the knowledge of the architect is defined as the [finished good] inventory, because this knowledge is sold to the client. The work-in-process inventory component could also exist for information flows. For instance, if the architect needs specific information about building codes, lack of which is preventing a movement to the next stage of the process, this secondary information flow acting as a work-in-process inventory is needed to be acquired to deliver the whole project.

Schaefers et al (2007) recently presented a more relevant paper in defining TOC’s performance measures for the health industry. They generalize the classical TOC's goal statement for publicly traded for-profit companies, namely "making money, both now and in the future", to be applicable to any type of institution, by rephrasing the goal as: "to continuously make more goal units today as well as in the future”.

To define the goal unit for a non-profit hospital, they take TOC's approach in that the goal unit should be the "value of the product or service in the eyes of the organization's customer". They claim that "the patient's objective is to receive the care that will maximize his life expectation with an acceptable to good life quality”, and as such meeting this objective should be the hospital's goal. Therefore, a measurement is needed that captures both life quality and life expectation. They formulate the goal unit (LETLIQ) as follows. 


$$
L E T L I Q=L E * L I Q
$$

where $L E$ represents the lifetime expectation (in years), and $L I Q$ represents the life quality (a factor between 0 and 1). They suggest units like QALY (and a number of others) as potential candidates for the LIQ factor, which we think is not correct since, for instance, the QALY encompasses both the life expectancy and the quality of life. We think they might have meant to suggest QALY (and the like) as the unit of measurement for LETLIQ.

Next, they formulate the goal unit earned for a particular patient through a medical act ( $A G U$ ) as:

$$
A G U=L E T L I Q_{o}-L E T L I Q_{i}
$$

where "o and i represent different values before and after the treatment". Our understanding is that they meant that index o refers to after the treatment and the index i refers to before the treatment.

If they mean the remaining LETLIQ at times $o$ and $i$ by the terms LETLIQ ${ }_{i}$ and LETLIQ $_{0}$, we think that their definition of AGU needs to account for the duration of the treatment as well. Two treatments of different durations with the same LETLIQ ${ }_{i}$ and LETLIQ ${ }_{0}$ have had different net effects on the level of LETLIQ, as more LETLIQ was lost just due to the passage of time during the longer treatment option.

If they mean the total (over the whole course of life) LETLIQ at times $o$ and $i$ by the terms LETLIQ $_{\mathrm{i}}$ and LETLIQ time. 
They define the health throughput of the hospital $(H T)$ for a given period of time $(t)$ as the number of actual accumulated goal units per unit of time, formulated as:

$$
H T=\frac{1}{t} \int_{0}^{t} A G U(\tau) d_{\tau}
$$

We think this is the average health throughput, and the throughput rate could be defined with a formula like:

$$
H T(\tau)=\frac{d}{d \tau} A G U(\tau)
$$

So the average formula still remains the same, but the equivalent of TOC's throughput has been also defined.

Next they discuss a difference between the hospital system and profit-oriented environments. They claim that industrial goal units should not meet any norms saying that for a specific transaction, the throughput should have a target value (as long as they respect ethical, legal, and environmental constraints). However, in the case of hospitals, the AGU must be compared to a standard or legitimate number of goal units that should be earned for a specific treatment, operation, or continuous care. They claim that "in most cases, the health specialist has been able to define quality levels to reach after having treated a patient who came in a given health status. This applies for life quality as well as for life expectation. It is obvious that legitimate values strongly depend on the patient's history”. This legitimate goal unit $(L G U)$ is then formulated as:

$$
L G U=L E T L I Q_{l}-L E T L I Q_{i}
$$


where the index $l$ refers to the legitimate value.

Similar to the case of AGU, depending on how the LETLIQ is defined, we think that their definition needs to either account for the duration of the treatment, or it should be made clear how the LIQ section is calculated during the treatment time.

They claim that comparing AGU and LGU allows measuring the quality and effectiveness of the system's output. Therefore, a quality index $(Q)$ is defined and formulated as:

$$
Q=A G U / L G U
$$

To formulate the inventory, they first generalize TOC's definition of inventory as "money absorbed in the system", to be applicable to any system by defining the inventory as: "all the goal units absorbed by the system”. They claim that health inventory is directly related to the period of time that a patient stays in the hospital or the total duration of the treatment.

They seem to be trying to capture the goal units absorbed in the system for which the throughput has not yet occurred as the level of inventory, but there are quite a few issues with their formulation. They formulate the inventory (presumable at any time $r$, but do not say this specifically) as:

$$
\left(L I Q_{l}-L I Q_{t}\right) \cdot t_{f}-\int_{t_{r}}^{t_{f}}\left(L I Q_{l}-L I Q_{r}\right)(t) d t
$$

where $i$ is initial, $f$ if final, and $r$ is current. 
It is not clear what the index $t$ is in the first term of the equation: $\left(L I Q_{l}-L I Q_{t}\right) \cdot t_{f}$. Moreover, it is not clear how two terms that do not have the same unit of measurement are being subtracted. And it is not clear why LETLIQ is not used instead of LIQ.

Also, the throughput is defined to exist only "when a patient definitely reaches the legitimate quality of life he was expecting from his treatment. This might never occur if, for any reason, the LIQ $Q_{0}$ remains below the legitimate level ". They do not specify how the health inventory, in case the throughput is not realized, is calculated. And once more, it is not clear why LIQ ${ }_{0}$, as opposed to LETLIQ $_{0}$, is discussed.

As for the operating expense, they keep the traditional TOC definitions. Besides the issues with the previous formulations, keeping the original TOC definition of operating expenses means that equivalent TOC measures of net profit and return on investment cannot be defined, since net profit is the subtraction of operating expenses from the throughput and in their formulation, the two have different units of measurement (LETLIQ/time unit and \$/time unit respectively). And return on investment only can be defined when the net profit has been defined.

\subsubsection{Thinking Process}

TOC's thinking process has been applied to a variety of settings such as supply chains (Rahman, 2002; Simatupang et al, 2004), police department (Taylor et al, 2003), manufacturing (Scoggin et al, 2003; Umble et al, 2006), strategic planning (Gupta et al, 2004), government sector (Shoemaker and Reid, 2005), and academic environments (Goldratt and Weiss, 2005). Besides the business novel by Wright and King (2006) that describes a hypothetical application of the thinking process in an NHS academic hospital (within the overall framework of the process of ongoing improvement), we found five papers reporting actual applications in the health care 
industry: Hunink (2001), Taylor and Sheffield (2002), Taylor and Churchwell (2003/2004), Mcnutt and Odwazny (2004), and Patwardhan et al (2006).

Hunink (2001) presents an interesting application of the thinking process to aid logical thinking and communicating about medical decision making. The author, a vascular radiologist, was frustrated by the vascular conference at the university hospital. The discussions on controversial patient cases went in a cycle of each person reiterating their point and evidence on what action to take. Taking the case of an elderly man with coronary artery disease who also had asymptomatic carotid artery stenosis, the question was whether a carotid endarterectomy should be performed prior to the coronary artery bypass surgery, or not performed at all. An evaporating cloud was built with the objective of increasing life and quality at acceptable costs by two requirements of (a) reducing stroke and mortality associated with CABG and (b) avoiding risks and costs of endarterectomy. The prerequisite to requirement (a) was an endarterectomy prior to CABG. The prerequisite to requirement (b) was no endarterectomy; hence a conflict. The assumptions underlying the relationship between the prerequisites and the requirements were examined and the supporting evidence from the literature were discussed in the meeting. The method focused the discussion and facilitated the communication among those involved with the care, and the best possible action (no carotid endarterectomy performed) was agreed on.

Taylor and Sheffiled (2002) applied TOC's thinking process to a Texas-based claims processing company that files, processes, and follows up medical insurance claims from local medical providers. Twelve UDEs were identified. A current reality tree was constructed that identified one of the UDEs (medical costs are growing faster than reimbursement levels) as the core problem. An evaporating cloud was built with the objective of achieving the opposite of the core problem, i.e. increasing the margin between reimbursement and cost. To this aim, claims 
should be entered correctly, and they should be entered promptly. The former requires taking time to properly training the staff; the latter requires putting new staff to work immediately. As such, there exists a conflict.

Five injections were considered: (a) add more edit checks to the system; (b) ensure that the claims processing entity receives EOBs; (c) provide additional staff training; (d) ensure that all valid claims are paid; and (e) ensure that claims are filed for the maximum legal amount. Finally, a future reality tree was developed to demonstrate the positive effect of these injections in addressing the UDEs.

Taylor and Churchwell (2004) apply the thinking process to tackle the problems of the General Medical Medicine (GMS) department of a Texas psychiatric state hospital. Within 24 hours of admission, patients should have a History and Physicals (H\&P) exam at the GMS department. Also, patients who develop medical conditions that require treatment are referred by the attending physician to the GMS department for a consult (evaluation and treatment). Patients might be sent back to the attending physician with recommendations for treatment, or be treated at the GMS. Severely ill patients are referred to outside medical services.

A total of eleven UDEs were identified. A current reality tree identified one of the UDEs (the decrease in the legislative funding) as the core problem. Two evaporating clouds were made, one to deal with the conflict between reducing workload and expenses and maintaining patient satisfaction; the other one to deal with the conflict between boosting nursing morale and increasing output. Injections were designed that, through a future reality tree, were shown to affect the hospital positively even if legislative funding decreases. 
McNutt and Odwazny (2004) discuss how the TOC provides the Patient Safety Committee of Rush University Medical Center with a conceptual framework for identifying core causes of error and offers the committee a model for planning safety improvements. The committee uses individual cases of serious adverse events, as opposed to trends or frequencies, to conduct an independent review of what has happened. First a chain of events leading to the adverse event, a timeline starting from the first discernible clinical decision and ending with the adverse event, is built. Despite the presence of multiple weak steps in the chain of events, the committee looks for a single step in the chain that is most likely responsible for the series of events that led to the adverse event. In investigating each case, the team develops a current reality tree by asking all possible logical questions to understand the causes. The tree pinpoints a single core cause. Next the steps are redrawn to see if a better way can be devised. Frequently, the core cause is a conflict, and that's why the team hears a lot of yelling and objections to their proposals.

Patwardhan et al (2006) present an application of TOC's thinking process to improve the process of developing technical reports for health care decision making in an Evidence-Based Practice Center. Created by the Agency for Healthcare Research and Quality (AHRQ), the objective of the EPC is to provide technical reports (in the form of evidence reports or technology reports) for clinical and public health policy makers (known as "partners").

Unused report was identified as the fundamental dominant UDE. The development of a current reality tree demonstrated four root causes and the core problem was identified as "partner does not know how to conceptualize and articulate needs, objectives, and specifications”, since it affects most of the other problems.

A set of five injections were considered, two of which (i) establish an ongoing relationship between partners, EPC, and AHRQ early in the process; and (ii) utilize a change clause, were 
deemed most useful in addressing the core problem. A future reality tree was then constructed that illustrated how the injections lead to the desirable effect of "Report is used".

\subsubsection{Critical Chain}

Some recent applications of the critical chain include construction projects (Yang, 2007) and repair and overhaul of aircraft in an air logistics center (Srinivasan et al, 2007). There is only one reported application of the critical chain to the health industry: Umble and Umble (2006).

Umble and Umble (2006) report the application of buffer management techniques to reduce Accident and Emergency (A\&E) department waiting times and the waiting times of acute hospital admissions of A\&E patients in three NHS facilities. Acknowledging that DBR is not applicable in this environment because it is not possible to schedule the arrival of patients, they take advantage of the buffer management techniques for project environments. Unlike most project environments where, due to the uniqueness of each project, there is a lack of feedback mechanisms to improve problematic processes for future projects, they identify the health care environment as a "highly repetitive multi-project environment" where buffer management concepts can be utilized to their full potential. Each patient is regarded as a new project, the progress of which, from patient arrival to discharge from A\&E or admission to acute beds, is monitored and compared against the performance standards set by the NHS. If there is reason to believe that the patient will not make it in time to meet the performance standards (e.g. patient is in the tracking or expedite buffer zones), then the resources are managed and the patient is expedited (if necessary) through the remaining steps to ensure the standards are met. Moreover, each week, a team of representatives review the sources of delays and focus their collective attention on improving the performance of the single activity that most negatively impacts waiting times. Statistical analyses of pre-implementation and post-implementation records 
demonstrate significant improvements with regards to the percentage of patients for whom the performance standards have been met.

\subsection{TOC for Publicly Funded Health Systems: Literature Gaps}

As discussed in section 2.2, we identified the drum-buffer-rope technique as a TOC tool that has not yet been applied to publicly funded health systems. Chapter three of this thesis makes a contribution to the application of the theory of constraints by applying the drum-buffer-rope technique to a publicly funded outpatient cancer facility.

While the drum-buffer-rope technique proved to effectively balance the tradeoff between the low-level performance measures in the specific case presented, assessing the benefits on overall health system performance proved to be difficult. This was mainly due to the fact that the equivalents of TOC’s system-wide goal and performance measures have not been adequately discussed in the published literature. Only Schaeffer et at (2007) provide a step in the right direction, but their proposed measurements fall short in providing the equivalent of TOC's system of performance measures. This is especially due to the fact that they define the throughput and inventory with units of life expectancy and quality, but keep the classical definition of operating expenses. Therefore, it is no surprise that they are unable to present the equivalents of cash flow, net profit, and return on investment, which are the ultimate global performance measures of the system. Chapter four of this thesis makes a theoretical contribution to the TOC field by developing the equivalent goal and performance measures for publicly funded health systems. 
Having defined the equivalents of TOC's system-wide goal and performance measures for publicly funded health systems, chapter five of this thesis provides a framework to link the lowlevel performance measures to system-wide measures, enabling to assess the effects of applying a specific tool of the theory, such as the drum-buffer-rope, with regards to the ultimate purpose of the theory which is to leverage system constraints to improve overall system performance. Extensive multi-disciplinary research is still required to accurately establish this linkage and thereby fully transfer the theory of constraints to publicly funded health settings.

\subsection{Main Thesis Contributions}

This section provides a brief overview of the contribution of each of the main chapters of this thesis to address one of the identified gaps in the literature of applying the theory of constraints to publicly funded health systems.

\subsubsection{Chapter 3: Drum-Buffer-Rope}

To explore the usefulness of the drum-buffer-rope scheduling technique in the health industry, a case study of its application to a publicly funded outpatient cancer facility is discussed in the third chapter of this thesis titled: "Drum-Buffer-Rope for an Outpatient Cancer Facility". With the use of a discrete event simulation model populated with historical data, the drum-buffer-rope scheduling policy is compared against "high constraint utilization" and "low wait time" scenarios. Drum-buffer-rope proved to be an effective mechanism in balancing the inherent tradeoff between the two performance measures of instances of delayed treatment and average patient wait time. To find the appropriate level of compromise in one performance measure in 
favor of the other, the linkage of these measures to system-wide performance measures, as developed in the fourth chapter of this thesis, are proposed. Finally, a variety of future research topics including a more intelligent sequencing of pre-booked patients at the constrained stage are recommended.

\subsubsection{Chapter 4: Goal and Performance Measures Development}

In reading the original TOC literature, it's hard not to notice how the goal and performance measures have only been developed for the publicly traded for-profit companies. This does not mean that the theory is not applicable to other settings. It does, however, imply that some customization would be required. Chapter four of this thesis, titled "Theory of Constraints' Performance Measures for Publicly Funded Health Systems”, addresses the key question of how to translate the traditional goal and performance measures to the environment of publicly funded health systems. To this end, a system dynamics representation of the classical TOC's systemwide goal and performance measures for publicly traded for-profit companies is developed, which forms the basis for developing a similar model for publicly funded health systems. The model is then expanded to include some of the factors that affect system performance, providing a framework to apply TOC's process of ongoing improvement in publicly funded health systems. Future research is proposed to more accurately define the factors affecting system performance and populate the model with evidence-based estimates for various parameters in order to use the model to guide TOC's process of ongoing improvement. 


\section{Drum-Buffer-Rope for an Outpatient Cancer Facility}

\subsection{Abstract}

To improve patient experience through providing more timely services in an outpatient cancer treatment setting, we proposed the adoption of a scheduling policy based on the theory of constraint's drum-buffer-rope (DBR) technique. With the use of a discrete event simulation model populated with historical data, we compared this policy against "high constraint utilization" and "low wait time” scenarios. DBR proved to be an effective mechanism in balancing the inherent tradeoff between the two performance measures of instances of delayed treatment and average patient wait time. To find the appropriate level of compromise in one performance measure in favor of the other, the linkage of these measures to system-wide performance measures, as developed in the fourth chapter of this thesis, are proposed. Finally, a variety of future research topics including a more intelligent sequencing of pre-booked patients at the constrained stage are recommended. 


\subsection{Introduction}

Acute leukemia patients usually go through a treatment path consisting of induction chemotherapy (3-4 weeks), restoration period (4-6 weeks), consolidation chemo (4-6 weeks), and (if possible and appropriate) bone marrow transplant. In the hospital under study, a highly specialized cancer facility, the consolidation chemo phase is administered on an outpatient basis for up to five times per week. Moreover, patients in remission still visit the hospital on an outpatient basis at least once every three months to ensure the disease has not reoccurred. The disease often recurs during a patient's lifetime and the patient may have to repeat the treatment path several times (usually up to three times) before receiving palliative care. As such, outpatient visits to the hospital (both during the consolidation chemo phase and remission) are a major part of an acute leukemia patient's experience of care and should be best managed by the hospital. This is no trivial task since patients only exit from the system through death or transfer to other hospitals and as such the volume of outpatients is on the increase over time.

The motivation for this study came from a common perception that even though the quality of clinical care received by outpatients in the hospital under study was excellent, there was room for improving the overall experience. Consequently, a multidisciplinary team of researchers with expertise in oncology, marketing, operations research, and change management came together to recommend strategies for a more patient centered model of care delivery. In phase one of this umbrella research study, patients and staff were interviewed to understand patient expectation and experience and the gap between the two. Besides a variety of qualitative issues that were discovered, there were concerns about the long wait times that patients had to endure during the 
day to complete their outpatient visit, sometimes to the extent that patients were asked to return the next day to complete their course of treatment (This event is referred to as "delayed treatment” through the rest of this chapter). Hence, the purpose of this research, which is the quantitative leg of the umbrella patient centered care research project, was defined to find strategies that would help the hospital best utilize its capacity to reduce average patient wait time and instances of delayed treatment for acute leukemia patients visiting the hospital on an outpatient basis.

This chapter is organized in the following order. First, a description of a typical outpatient visit and some of the uncertainties that hinder proper advance planning are described. Next, the fundamentals of the theory of constraints and its drum-buffer-rope logistical technique to control flow as well as some relevant literature are introduced. Subsequently, a discrete event simulation of the current outpatient processes populated with historical data is described and validated against some patients' self collected data. The model is then used to compare the recommended drum-buffer-rope based scheduling policy against "high constraint utilization” and "low wait time” scenarios with regards to the two performance measures of instances of delayed treatment and average patient wait time. Next, we discuss some observations and make recommendations on record keeping practices at the constrained resource and conclude with proposing a number of areas for future research including a more intelligent sequencing of patients at the constrained stage. Finally, to find the appropriate level of compromise in one performance measure in favor of the other, the linkage of these measures to system-wide performance measures, as developed in the fourth chapter of this thesis, are proposed. The fifth chapter of the thesis provides a framework to establish such a linkage. 


\subsection{Outpatient Visit}

A typical leukemia outpatient visit consists of a number of stages as schematically shown in figure 3.1. Starting from the left, patients arrive at the hospital to have their blood drawn. For leukemia patients, the majority of whom have a central Hickman line (a special intravenous device used to give fluids intravenously), the blood is drawn in the transfusion unit department (TFU). The specimens are then sent to the blood lab for analysis. Meanwhile, patients with a clinic appointment or drop-in patients who have come to the hospital to get a physician consult (usually because they are feeling extremely sick) wait in the waiting area outside the clinics to be seen by their physician.

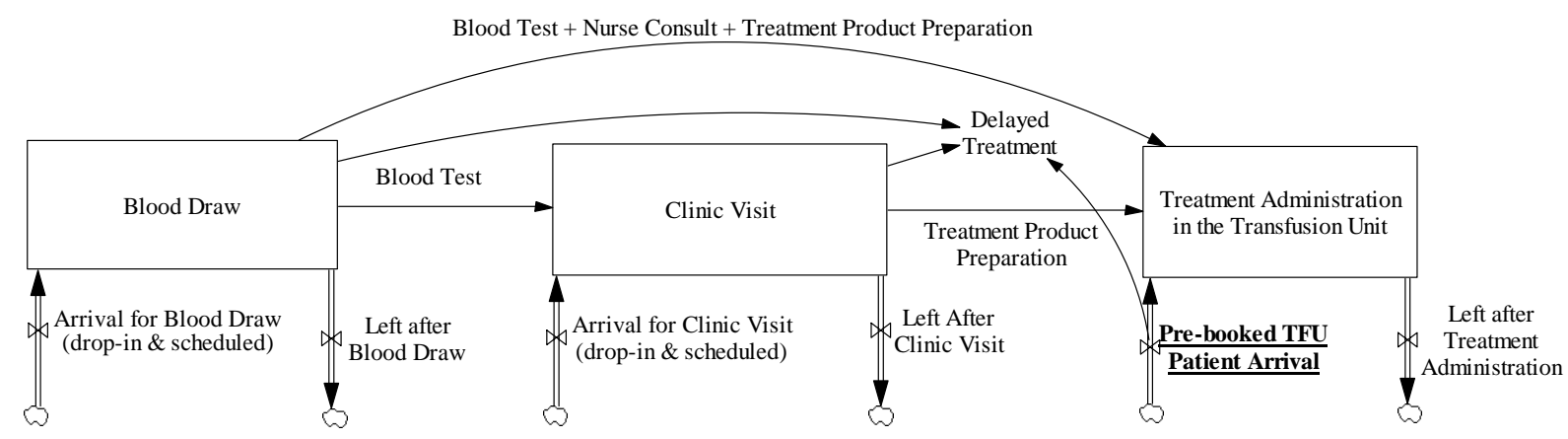

Figure 3.1: Typical Outpatient Visit

Some patients bypass the clinic visit stage and go directly to the transfusion unit. These are usually patients whose blood test results are reviewed by the highly trained advanced practitioner nurses in the transfusion unit. Moreover, patients who have their blood drawn prior to their visit (e.g. on the day before) or outside the hospital (at a collaborating laboratory in their community which makes the results available to the hospital) may come directly to the clinic waiting area. 
Once the patients are seen by the physician in the clinic or an advanced practitioner nurse in the transfusion unit (for those patients who bypass the clinic stage), an appropriate treatment (blood transfusion, chemotherapy, or antibiotics) may be ordered for them. Patients who do not require any treatments leave the hospital. The rest would wait outside the transfusion unit (where all types of outpatient treatments for leukemia patients are administered) while their treatment product is being prepared either in the blood bank (for transfusion products) or the pharmacy department (for antibiotics and chemotherapy products). Ideally, the patients then receive their treatment in the transfusion unit. However, it is possible that by the time the treatment is ready there are either no treatment chairs available in the transfusion unit or not enough operating hours left in the day to administer the treatment, especially since these treatments could take several hours to administer. In such cases, the patient will receive a delayed treatment: the patient is asked to leave the facility and return the next day to receive the treatment.

Finally, the pre-booked TFU patient group, who have a scheduled appointment at the transfusion unit and do not require blood work (e.g. those on a chemotherapy regimen pre-ordered by a physician for a set number of days), show up directly at the transfusion unit. Ideally, the treatment product for these patients has been prepared before their arrival, as their arrival and their required treatment product were known in advance. It is still possible that these patients receive a delayed treatment as well, for instance if all the remaining treatment chairs are occupied by more emergent drop-in patients. These pre-booked TFU patients are the patient group whose rate of arrival can be scheduled in advance and, as shown later in this chapter, they go through the main constrained stage of the system. As such, they are the target of the drumbuffer-rope based scheduling policy. 


\subsection{Problem Characteristics}

There are a number of characteristics that make it difficult to plan the operations in advance. First, the treatments for most patients (all but the pre-booked TFU patient group) are not known in advance and depend on the patient's health status which could vary on a daily basis. This means that until a blood test is done and the results are reviewed, the type and length of the treatment (if any) that the patient would be receiving is not known. Moreover, since each treatment product is customized to an individual patient's health status, it's not possible to keep a stock of different treatment products to be used by any patient who might need them.

Hence, certain types of waits (e.g. waits for blood test results or treatment product preparation) and instances of delayed treatments arising from them are due to medical reasons and as such inevitable. However, logistics delays, mainly those related to unavailability of resources (e.g. waits for treatment chairs in the transfusion unit) and instances of delayed treatments arising from them can be potentially reduced through better planning.

These special characteristics, however, are not unique to this environment. In fact, they are very similar to a remanufacturing environment where "repair of any product is subject to randomness on many levels, and part mixes are probabilistic because what part needs repair is not known until the product is induced, disassembled, and inspected. In addition, operation routeings are dependent on the nature of the repair required, and the processing time required at each step of the assigned routeing is also a random variable. The processing time required at any given operation is a function of the degree of wear of the part and the operating conditions to which the

part has been subjected.” (Guide, 1996). Thus, given the demonstrated effectiveness of theory of constraint's drum-buffer-rope (DBR) scheduling technique in the latter setting (Guide, 1996), 
there is reason to believe that DBR could be of benefit to better plan the operations in the hospital under study. The choice of DBR is also in agreement with Siha's (1999) suggestion that DBR can be useful in medical environments with an interaction of stochastic cure times, dependent events, and probabilistic care plans.

To set the context before examining the usefulness of DBR in the setting under study, first the fundamentals of the theory of constraints (TOC) and its DBR scheduling technique following by some relevant literature are introduced in the next sections.

\subsection{Fundamentals of TOC and DBR}

First introduced by Goldratt (Goldratt and Cox, 1984), theory of constraints views systems and processes as series of dependent events. Like the fact that a chain is only as strong as its weakest link, all systems have at least one and at most a few constraints or bottlenecks which limit system performance (Womack and Flowers, 1999). At an aggregate level, TOC’s management tools can be categorized into three branches: TOC's process of ongoing improvement (the goal, performance measures, and the five focusing steps), logistics (drum-buffer-rope and buffer management techniques), and problem solving/thinking tools.

TOC’s process of ongoing improvements fundamentally consists of two prerequisites for implementation, defining the goal and performance measures of the system, and five focusing steps: First system constraints are identified. Second, they are exploited by making physical constraints as effective as possible and eliminating policy constraints. The third step is to subordinate the system to the constraint. If the system performance is not enhanced to the desired 
extent, in the fourth stage the constraint is elevated by increasing its capacity. Recognizing the fact that the system constraint has probably shifted, the final stage is to start over from the first step and identify the new system constraint. (Rahman, 1998)

Logistically, the implementation of TOC for physically-constrained systems is governed by the drum-buffer-rope (DBR) technique, explained in detail by Goldratt and Fox (1986). DBR "synchronizes resources and material utilization in an organization" to ensure resources and material are used "only at a level that contribute to the organization's ability to achieve throughput” (Rahman, 1998). Consistent with the focusing step one, the constraint of the system is identified which sets the pace (drum beat) of the system. Exploiting the constraint in the second focusing step is performed by strategically placing buffers. Finally, consistent with focusing step three, the rope is the mechanism to subordinate non-constraint stages by releasing inventory to the system in accordance with the drum beat. (Watson et al., 2007)

There is a tradeoff between the level of the protection of the constraint (as demonstrated by the buffer size) and lead times. Protecting the constraint through increasing buffer size necessitates releasing material earlier, which increases both work-in-progress and lead times (Watson et al., 2007). Buffer management is a related TOC application that determines the size and monitors the status of buffers. Determining the appropriate level of buffers is an ongoing research area; for instances, see Radovilsky (1998), Gunn and Nahavandi (2000), Louw and Page (2004), and Ye (2007).

There are two main types of buffers. Time buffers, physically represented by the amount of work-in-progress inventory (Watson et al., 2007), protect the constraint from disruptions at nonconstraint resources (Rahman, 1998). Capacity buffers (extra capacity at non-constraints) make it possible to maintain time buffers "in times processes experience fluctuations in output" (Watson 
et al., 2007). There is a tradeoff between the requirements for the two main buffer categories, as stated by Blackstone and Cox (2002): “All that can be said definitely is that there is a tradeoff between protective inventory and protective capacity and that a threshold level of protective inventory is needed to transmit the protective capacity and vice versa.”

In practice, buffer levels can be set to some initial levels, for instance according to the rules of thumb mentioned in Ricketts (2008). Over time, buffers can be monitored and their right level can be determined through experience. Additionally, by comparing actual versus planned buffer sizes at set times, management can identify problems before they become critical, and through early feedback to the problematic process(es) reduce the need to expedite. Improvement efforts can then be focused on the more problematic processes. Monitoring buffers also makes it possible to assess the impacts of improvement initiatives as they are being implemented. (Watson et al., 2007)

Goldratt (1997) introduced buffer management techniques applied to project environments separately from DBR. Emphasizing the fact that the goal is to complete the project on time, not individual activities, most of the excess time allocated to each activity is taken away to create a buffer for the whole project. This project buffer, which acts as the "final shock absorber for all of the delays on the critical chain and other non-critical feeding paths” (Umble and Umble, 2006), is monitored throughout the project. The buffer is usually divided to three zones: safety, tracking, and expedite zone. A project that has not been completed yet is referred to as a hole. Holes in the safety zone are considered natural. When a hole reaches the tracking zone, it is investigated. But no action is taken until the hole reaches the expedite zone, meaning that the delays have depleted the project buffer to the extent that project completion time is jeopardized. At this point, management actions such as reassigning resources are implemented. This comes at no surprise to 
management since the delays have already been noticed and investigated when the hole was in the tracking zone. The feedback mechanism present in buffer management in non-project environments, where improvement efforts are focused on problematic processes for the sake of improving future performance, is generally absent in the project environment because by their definition, projects are one-time unique sets of activities. (Umble and Umble, 2006)

Finally, the third branch of TOC is the problem solving/thinking tools, mainly targeting policy constrained systems. In fact, Motwani et al (1996) claim that most constraints in organizations are policy or procedural constraints, such as operational directive forbidding overtime, rather than physical. The tools of thinking processes including current reality tree, evaporating cloud, future reality tree, prerequisite tree, and transition tree are well established and can be found in a lot of sources, including Goldratt (1994) and Dettmer (1997).

\subsection{TOC in Health Care: A Review of Literature}

Unlike the huge literature on applying TOC in manufacturing, as partly reviewed by Mabin and Balderstone (2000), there are relatively few published applications in health care. Lubitsh et al. (2005) assess the impact of TOC implementations on NHS Trust departments of neurosurgery, eyes, and ENT. Most other studies describe the application of the first and last branch of TOC, namely the process of ongoing improvement and problem solving/thinking tools, to settings different than ours including primary care (Womack and Flowers, 1999), medical decision making (Hunink, 2001), emergency departments (Rotstein et al., 2002), medical insurance claims processing (Taylor and Sheffiled, 2002), psychiatric hospitals (Taylor and Churchwell, 2004), 
reduction of medical errors (McNutt et al., 2004), evidence-based practice centers (Patwardhan et al., 2006), and community mental health centers (Gupta and Kline, 2008).

Kershaw (2000) applied the TOC’s process of ongoing improvement to a setting very similar to ours. The study applied the focusing steps to an outpatient oncology clinic administering chemotherapy to patients. The process is composed of six steps: checking in with the receptionist, blood tests in the lab, pretreatment process conducted by nurses in the exam room, physician consult in the exam room, chemotherapy administration in the treatment room, and scheduling a follow-up appointment with the receptionist. To identify the bottleneck, patient volumes were compared to the capacity of resources at each step of the treatment process and the treatment chairs were found to be the primary constrained resource. To let the constraint set the pace, patients were scheduled based on constraints' capacity, which necessitated unacceptable hours of overtime. Thus, improvement efforts were focused on the constraint. First, the three components of the treatment time- establishing intravenous access, administering prescribed drugs, and performing post treatment education- were analyzed. It was decided that the post treatment education could be performed during drug administration and that the intravenous access, for more routine patients, could be established in the blood lab by lab technicians. Moreover, mobile supply caddies were set up so that time was not lost due to nurses looking for equipment and supplies when patients experienced undesirable side effects. At the end of the study, the capacity was increased by 20 to 25 percent.

Umble and Umble (2006) report the application of buffer management techniques to reduce Accident and Emergency (A\&E) department waiting times and the waiting times of acute hospital admissions of A\&E patients in three NHS facilities. Acknowledging that DBR is not applicable in this environment because it is not possible to schedule the arrival of patients, they 
take advantage of the buffer management techniques for project environments. Unlike most project environments where, due to the uniqueness of each project, there is a lack of feedback mechanisms to improve problematic processes for future projects, they identify the health care environment as a "highly repetitive multi-project environment" where buffer management concepts can be utilized to their full potential. Each patient is regarded as a new project, the progress of which, from patient arrival to discharge from A\&E or admission to acute beds, is monitored and compared against the performance standards set by the NHS. If there is reason to believe that the patient will not make it in time to meet the performance standards (e.g. patient is in the tracking or expedite buffer zones), then the resources are managed and the patient is expedited (if necessary) through the remaining steps to ensure the standards are met. Moreover, each week, a team of representatives review the sources of delays and focus their collective attention on improving the performance of the single activity that most negatively impacts waiting times. Statistical analyses of pre-implementation and post-implementation records demonstrate significant improvements with regards to the percentage of patients for whom the performance standards have been met.

To the best of our knowledge, this study is the first to apply DBR scheduling technique to the health industry. The studies by Kershaw (2000) and Umble and Umble (2006) can both be seen as complementary to this study. A number of the bottleneck exploitation strategies described by Kershaw (2000), such as delegating the responsibility of establishing intravenous access for more routine patients to lab technicians and conducting the post-treatment education during the last minutes of treatment administration, are strategies that can potentially improve the performance of the bottleneck resource (treatment chairs in the transfusion unit, as discussed later in the chapter) in the cancer facility under study as well. This study assumes that such performance improvement initiatives at the bottleneck have already been accomplished and 
explores ways to further exploit the constraint by determining the appropriate (time) buffer sizes to ensure high constraint utilization and subordinate the system to the constraint by scheduling the pre-booked TFU patient group according to the drum beat. Umble and Umble's (2006) buffer management strategies could complement this study for tracking the progress of different patient groups to ensure meeting pre-set served-within-time-limit standards, should such standards be set by management or other authorities.

\subsection{The Simulation Model}

We constructed a discrete event simulation model that describes the operation of the leukemia outpatient treatment facility during weekdays (Monday to Friday) based on historical data analysis, patients' self-track results, and expert opinion. The model comprises the typical outpatient visit stages: blood collection, blood test, clinic visit, transfusion product preparation in the blood lab, chemotherapy or antibiotics product preparation in the pharmacy, and treatment administration in the transfusion unit. We implemented the simulation model using the Simul8 software.

While the whole structure of the model is the same for all days of the week, a number of model parameters (e.g. number of clinics open in the morning or afternoon of each day) and the input data (e.g. percentage of outpatients following a specific route) differ by day of the week. There is no warm up period for any of the days, meaning that similar to the real world situation, the outpatient facility starts from zero patients present at the hospital at the start of each day. The simulation period for each run of each day of the week was set to the length of a full day. 
To ensure that different scheduling policies are compared under similar experimental conditions, common random numbers (Law, 2007) were used. To allow this, all patients are entered into the model (in a dummy queue) at the beginning of the day (before the actual hospital operation hours) and assigned a number of labels that define the stages that they go through over the course of the day (the patient route) and the processing time or turn-around time at each stage.

For stages that occur in facilities dedicated to leukemia patients for the most part (blood collection in the transfusion unit, clinic visit, and treatment administration in the transfusion unit), the processing time is used. The processing time is defined as the length of time the patient spends with the most constrained resource of a specific stage. It does not include the wait times associated with that stage or the time spent with less constrained resources. For instance, even though the clinic visit stage consists of a number of steps including patients signing in with the receptionist, nurses checking for the availability of patients' blood test results in the computer system, nurses or volunteers taking the weight of the patients, admittance to the consultation room, receiving consultation from the physician, and the waits in between these steps, only the consultation time with the physician was modeled as the processing time of the clinic visit stage. The other steps are either performed for operational reasons and as such are not medically necessary (such as signing in with the receptionist), or are medically necessary but performed by resources that, if need be, are not difficult to increase (such as having the vital signs taken). Since the model aims at finding the overall constraint of the whole system, we decided that the less constrained steps within each stage along with all the internal waits in between the different steps of a stage in an outpatient visit can be all categorized as the wait times at that stage for the purpose of this study. In fact, these steps are all wait times to get to the real value-added step of physician consult. Hence, for the clinic visit stage, only the time spent with the physician is modeled as the processing time. 
Similarly, for the treatment administration stage at the transfusion unit, only the portion of time that the patient is occupying a treatment chair (as the most constrained resource in the transfusion unit) is considered as the processing time. All other steps and the wait times in between the steps of receiving treatment in the transfusion unit are considered as wait times in the simulation model. The time occupying a treatment chair is also a good proxy of the other constrained resource in the transfusion unit, nurse availabilities, since a constant nurse-to-chair ratio is maintained in the unit. Finally, for the blood collection stage, only the time spent with the most constrained resource, the nurse drawing the blood, is considered as the processing time.

For stages that occur in facilities shared by a larger patient population (blood test in the blood lab, transfusion product preparation in the blood bank, and chemotherapy or antibiotics product preparation in the pharmacy) the turn-around time is used. Turn-around time is the door-to-door time spent at a stage, which includes the processing time of that stage plus the wait times associated with that stage. The reason for the choice of turn-around times is lack of access to required data to correctly model these stages. In order to model the resources shared with nonleukemia patients, the demand placed on these resources from non-leukemia patients should be known and modeled as well. We did not have access to this data, and as such had to use the turnaround times as the input to the model.

Once the operating hours start, the patients, who already have been assigned labels of patient route and processing or turn-around times at different stages, are entered into the first stage of their visit according to the assumed arrival pattern (for arrivals at the blood collection and clinic visit stages, as described in the next section) or the scheduling policy being used for the specific scenario (for pre-booked TFU patient arrivals). With this common random numbers approach, different scenarios are compared with regards to exactly the same patients in each run of the 
simulation model, and as such any differences in their comparative performance can be attributed to their structural differences, as opposed to differences in random events.

\subsection{Data Needs, Sources, and Assumptions}

Six categories of input data are needed to run the simulation model for each day of the week: patient route probabilities, patient volumes, processing times or turn-around times at different stages of the outpatient visit, distribution of patients among various clinics of the day, number and operating hours of the resources, and patient arrival times at the hospital.

Patient routes were entirely derived from historical data analysis. After being granted proper ethics approvals, the list of MRNs or medical record numbers (unique patient identifiers at the hospital under study) of all acute leukemia patients newly diagnosed between Jan 1, 2005 and June 30, 2005 (a total of 87 MRNs) was obtained from the Cancer Registry department of the hospital. Subsequently, patient activities (both inpatient and outpatient) between Jan 1, 2005 and Mar 1, 2006 of the above MRNs were electronically retrieved from the four different information systems at the hospital and combined together using the unique MRNs of patients. A data cleaning task was performed on the massive volume to records to separate the inpatient and outpatient pieces of data, as only the latter was of interest to this research. Specifically, the outpatient data pieces included date and time stamps of the following activities: specimen collection, specimen received, result entry, summary result review, clinic visits, transfusion product availability, chemotherapy or antibiotics product availability, and transfusion unit visits. 
While the initial goal was to use the time stamps to recreate the patient routes and calculate the turn-around times of different units (to validate the model against it), upon receipt of the data, the time stamps appeared to be of poor quality. For instance, there were numerous instances that almost all patients were checked into the clinic at the same time, hinting that the unit secretary probably signed in all scheduled patients at the start of the day to "save time" later. There were also instances of blood results availability time stamps before the specimen collection time stamps. Therefore, only the units visited, as determined by the pieces of data available, were used to generate the patient routes. Given the inaccuracy of the time stamps, the direction of the movement was assumed to be always from the left to the right of the following stages: blood collection, blood test, clinic, blood product or chemotherapy/antibiotics product preparation, and treatment administration.

Patient volumes (for the 2005-2006 fiscal year) or how many patients visited each of the units were obtained from the activity reports of the hospital. Combining patient volumes at each unit and patient route probabilities, we were able to calculate back the average number of patients who arrived at each of the units from outside the hospital. The simulation model took the reverse direction: simulating the path of patients after arrival was accomplished by using the calculated average number of patient arrivals at each of the units and the historical patient route probabilities. The only major roadblock in doing so was deciding which clinics the patients belonged to. On each specific day, there are a number of morning and afternoon clinics operating. Patients normally belong to a specific clinic and are supposed to visit their own clinic under normal circumstances. Anecdotally, we were told that the patient load is not distributed equally among the clinics and some are more crowded than others. However, we were unable to get statistics on the distribution of patients to different clinics within one day. As such, we assumed an even distribution of patients to clinics. While at first this seems a simplistic 
assumption, it actually serves very well the purpose of the model: to find the physical bottleneck of the system and subordinate the system to it by using the DBR technique. If one clinic appears to be the bottleneck when another one on the same shift is not, then the real bottleneck is not the capacity of the clinics (i.e. the physicians' time) but rather a policy constraint with regards to the distribution of the patients to the clinics. TOC's recommendation for policy constraints is to eliminate them, rather than subordinating the system to them. So, by and large, the assumption of equal distribution of patients to different clinics does in fact serve the purpose of the model.

As for the processing times, where appropriate, the findings of the patient self-track forms which were distributed to consenting patients to track the stages of their visits during the day was inputted to Statsfit software package to generate appropriate statistical distributions. Where there were an inadequate number of self track forms involving a specific activity, expert opinion was sought. Turn-around times of stages occurring in non-leukemia dedicated units were acquired from internal hospital reports.

The number and operating hours of the most constrained resources in each stage of the outpatient visit (i.e. nurses for blood draws in the transfusion unit, physicians for consults in the clinic, and treatment chairs for treatment administration in the transfusion unit) were obtained from the hospital contacts.

Unfortunately, we were not able to obtain patient arrival times to generate arrival patterns. The scheduling system appeared to be missing the scheduled times (as a proxy of patient arrival times) for a considerable number of patients. According to the terms of the ethics approval, we were not allowed to talk to patients. Without talking to patients, even observing patient arrivals at different stages (except for the blood collection stage) is not useful for one does not know whether or not this is the first unit they are arriving at (e.g. whether an arrival to the clinic is 
from outside the hospital, as opposed to the blood collection stage). The number of self-track forms received was not adequate to make assumptions about patient arrival patterns either. To run the model, however, we had to assume some arrival pattern for each of the stages where patients can possibly arrive at (from outside the hospital): blood collection, clinic visit, and treatment administration at the transfusion unit.

We sought expert opinion to make our assumptions about patient arrival patterns as realistic as possible. We were told that most patients arrive at the hospital to have their blood drawn early in the morning, in the hope to get in front of the queue at the consequent stages. This was supported by our limited observations at the department. As such, in our model, we assume all patients who need blood work arrive at the start of the shift. As for the clinics, we observed that patients were scheduled throughout the shift. Though patient arrivals do not necessarily match patient appointments, we had no better clue than the scheduling policy and as such we assume a constant arrival rate for patients during the regular clinic hours. Clinics usually go overtime (both in the real world and in the simulation model), but patients are assumed to arrive during regular clinic shifts.

Unfortunately, we were not able to make any assumptions about the arrival pattern of the prebooked TFU patient group to receive (pre-ordered) treatments in the transfusion unit. As later discussed in this chapter, we discovered that the treatment chairs in the transfusion unit were the major bottleneck of the system, and as such the DBR strategy mainly aims at exploring ways to best schedule the arrival of pre-booked TFU patient group so that the constraint is highly utilized while keeping average patient wait time in a reasonable range. As such, the ideal patient arrival pattern at the TFU is the outcome that this research is seeking to produce and the lack of access to the current practice does not severely limit the research. However, the lack of a basic 
assumption for the current patient scheduling practice at the TFU does limit our capability in fully validating the model against the real world. Fortunately, a partial validation of the model is still possible and should suffice to ensure the model is realistic enough to explore scheduling policies at the transfusion unit.

\subsection{Model Output and Validation}

The model produces a number of output metrics, such as wait times, number of patients waiting, and resource utilization at each stage of the outpatient visit. We use the one output metric for which we received some real world data through patient self-track forms, namely average patient wait time at each stage, for model validation. We only have 5 self track forms to validate the model against, so we confess that this is a very soft validation of the model.

Since we do not have patient arrival patterns for the transfusion unit (for those patients coming to the transfusion unit from outside the hospital, i.e. the pre-booked TFU patient group), we cannot validate average patient wait time at the transfusion unit stage against the received data. Fortunately, since the treatment administration at the transfusion unit is the last stage of the outpatient visit, the average patient wait times at the previous stages are not affected by it and as such can be validated without regards to the validity of the value of the average patient wait time at the treatment administration stage.

We decided to use the simulation model to build a confidence interval with a specified precision of at least 5 minutes. The choice of 5 minutes was informed by the fact that patients appeared to have rounded the time stamps to minutes ending in 0 or 5 (e.g. 9:25 or 9:30). Using the confidence intervals with specified precision method as described by Bank et. al. (2005), we calculated that the number of runs required to build a confidence interval at a precision of 5 
minutes for the average patient wait time at the blood collection, morning clinic visit, and afternoon clinic stages were 72, 35, and 81 respectively. Table 3.1 compares the mean of each output metrics derived from the self track forms to the mean and 95\% confidence intervals of the simulated output metric acquired by running the model for the required number of replications to get the specified precision.

Please note that the actual morning and afternoon average patient wait times reported in table 3.1 are for patients who have had their blood drawn prior to the clinic visit, as this was the case for the sample of self track forms that we received. To compare corresponding values together, the simulated average patient wait time for morning and afternoon clinic visits reported in table 3.1 is indeed the sum of the blood test turn-around time and the wait time for the clinic visit.

It is seen from table 3.1 that the real-world estimates of average patient wait time for blood collection and afternoon clinic visit fall within the 95\% confidence interval of the simulated output measures. However, this is not the case for the average patient wait time for the morning clinic visit, as the real world estimate appears to be higher than the $95 \%$ confidence interval of the simulated results. We speculate this difference stems from the fact that, in our simulation model, the blood lab is only modeled at an aggregate level with its statistical distribution of the turn-around time. The statistical distribution used is based on expert opinion and does not differentiate between morning and afternoon shifts. However, from our limited observations, it appears that mornings are especially busy at the blood lab and as such the turn-around times should be relatively higher. So the simulation model might be assuming an under estimate of the blood lab turn-around times during the morning shift; Added to the wait time for the physician, the estimate of the total wait time from patient's perspective at the morning clinic visit stage 
might be underestimated in the model compared to the real world. We will discuss how this might have affected the findings of this research under section 3.14.

\begin{tabular}{|l|l|l|c|c|}
\cline { 3 - 5 } \multicolumn{2}{c|}{} & Actual & \multicolumn{2}{c|}{ Simulation } \\
\hline Outpatient Visit Stage & Metric & Mean & $95 \%$ CI & \# of runs \\
\hline Blood Collection & Average Wait Time & 29.3 & {$[28.10,33.12]$} & 72 \\
\hline Morning Clinic Visit & Average Wait Time & 131.4 & {$[119.82,125.01]$} & 35 \\
\hline Afternoon Clinic Visit & Average Wait Time & 131.4 & {$[127.52,132.54]$} & 81 \\
\hline
\end{tabular}

\section{Table 3.1: Model Validation}

Although we do not have real world estimates for other output metrics (e.g. average number of people waiting and resource utilization at each stage of the outpatient visit), we agree with Santibañez et al. (2008) that when the other output metrics are inter-related to those we were able to successfully validate against the real world data, it could indicate that other metrics are reasonably valid as well.

Now that the model output metrics have been validated to the extent possible, we can define the performance measures that we will use in comparing the two scheduling policies against each other. 


\subsection{Performance Measures and Scenario Definitions}

We chose two performance measures that best demonstrate the tradeoff between protection of the constraint and lead times: instance of delayed treatment and average patient wait time. We hypothesize that the earlier patients are scheduled, the more the constraint is protected from starvation. As a result, the constraint is better exploited, enabling it to serve more patients, leading to fewer instances of delayed treatment. However, early scheduling of patients would deteriorate average patient wait time. To test our hypothesis, we use our discrete event simulation model to compare three scenarios:

High constraint utilization: We explore a constraint protective scenario in which patients are scheduled to be at the constrained stage as soon as there is a slight chance that the constrained resource can serve them, but not earlier.

Low wait time: We explore a wait time protective scenario in which patients are scheduled late enough to ensure it is highly likely that the constrained resource can serve them right away, but not later.

Drum-buffer-rope: We explore a more balanced scenario that maintains a low average patient wait time without significantly increasing instances of delayed treatment. Patients are scheduled to be at the constrained stage at times when there is a fair probability that the constrained resource is available to serve them.

The key, of course, is determining when patients should be scheduled to be at the constrained resource stage in each of the scenarios. In section 3.12, we will describe how we used our 
discrete event simulation model to answer this question. But first, the constrained resource or the bottleneck of the system should be identified.

\subsection{Finding the Bottleneck}

As discussed earlier in this chapter, the first step of TOC's process of ongoing improvement is to find the bottleneck. This is the prerequisite for the drum-buffer-rope technique as well, as the bottleneck sets the drum beat. Unfortunately, the theory of constraints literature is not very clear on how to find the bottleneck. While in some manufacturing systems, such as the one depicted in the Goldratt and Cox (1984), visual inspection of the facility helps in identifying the bottleneck, this is not always the case. First, in environments like health care where there is a parallel flow of patients, information, and material (Sale, 2005), it is not obvious by observation where the real congestion is. For instance, in the setting under study, patients may be waiting outside the clinic, but this may or may not mean that physicians are the constraint. Patients might indeed be waiting for blood test results (information flow). Similarly, a high number of patients waiting outside the transfusion unit does not necessarily indicate insufficient treatment chair capacity in the unit, as patients might be waiting for various treatment products (material flow). Second, as Fung (1999) demonstrated, in a mixed push/pull system, the location of the slowest link does not generally coincide with where the greatest congestion is. As such, one has to think of a more robust method to find the bottleneck.

We chose to use the simulation model to pinpoint the constraint. We ran the simulation model for 2500 runs, which is above the recommended 2042 runs by the trial calculator feature of the Simul8 software package for a 5 percent confidence limit around the estimate of the mean of the 
selected simulation results. We compared the daily demand for blood collection nurses' time, physicians' time, and TFU treatment chairs, against their daily capacity. For all days except Mondays, TFU treatment chairs were identified as the bottleneck with the highest demand to capacity ratio. On Mondays, physician time was the bottleneck. This location of the constraint was not sensitive to slight changes in the estimated processing times at the blood collection, clinic visit, and treatment administration at the transfusion unit stages.

We chose to analyze the effectiveness of the drum-buffer-rope based scheduling policy for one of the days when the TFU treatment chairs were the bottleneck, as opposed to the case of physicians' time constrained Mondays. The reason was two-fold. First, we did not have data on which of the patients arriving at the clinics from outside the hospital are scheduled patients, the arrival of whom could possibly be affected through a drum-buffer-rope based scheduling policy, as opposed to drop-in patients (who are feeling sick and show up at the hospital at their own discretion). Second and more important, while physicians' time is indeed the bottleneck on Mondays, it is more of a policy constraint as opposed to a physical constraint. Using the simulation model to estimate the demand for a whole week demonstrates that the ratio of total demand to total capacity for physicians' time is less than that of the treatment chairs in the TFU unit. As such, should the clinics (i.e. number of physicians practicing each day) be distributed more evenly throughout the week or should the patient volumes be more evenly redistributed to clinics on different days of the week, this constraint would disappear. This finding is in fact similar to the recommendation by Santibañez et al. (2008) who also suggested redistributing the clinic workload more evenly across the week and time of day when analyzing one of the British Columbia Cancer Agency's Ambulatory Care Unit. We are aware of potential resistance to change, especially from the physicians who are used to their current schedule and possibly from patients who might dislike or fear discontinuity of care as a result of being reassigned to new 
physicians. However, as discussed earlier, the TOC literature emphasizes the importance of eliminating policy constraints and introduces a number of useful tools to facilitate that (Goldratt, 1994). Since the focus of this research is on exploiting and subordinating the system to the physical constraint by using the DBR technique, we concentrate on developing the details of the DBR based scheduling policy.

Among the remaining days that the TFU treatment chairs were identified as the bottleneck (Tuesday-Friday), we chose to analyze the case of Wednesdays as it possessed both morning and afternoon clinics (and the most number of each) and could demonstrate the effectiveness of the policy in a more complex environment. The methodology used, however, can be applied to other days of the week as well.

\subsection{Scenario Development}

As mentioned in section 3.10, we compare three scheduling scenarios to schedule the pre-booked TFU patient group: high constraint utilization, low wait time, and drum-buffer-rope. In this section, we discuss how we modeled each of the scheduling scenarios in our discrete event simulation model.

\subsubsection{High Constraint Utilization Scenario}

In order to prevent the constrained resource from starvation and thereby maintain low instances of delayed treatment, it is sufficient to schedule patients to arrive at TFU when there is the slightest chance that there is a treatment chair available for them, but not earlier. Given the stochastic nature of the various input parameters of the model, it is very difficult to analytically 
calculate the treatment chair availability times. As such, we used the simulation model to estimate the treatment chair availability times. We first ran the model with a significantly large number of the pre-scheduled TFU patients entering the queue for the TFU treatment chairs at the start of the operating hours, but gave this patient group a lower priority in taking the TFU chair when compared to those who were coming from within the facility (either from the clinic visit or from the blood collection stage). The model recorded the times that the pre-scheduled TFU patients were assigned to a TFU treatment chair. These times were indeed the times that, in absence of a pre-scheduled TFU patient, a TFU treatment chair would have been left idle. If this was not the case, a non pre-booked TFU patient would have had a higher priority to take it. The results of these time logs for 2500 runs were exported to Excel and the minimum time that the $\mathrm{n}^{\text {th }}$ pre-booked TFU patient was assigned to a treatment chair was used as a time estimator of when there is a slight chance that a chair is available should the $\mathrm{n}^{\text {th }}$ pre-booked TFU patient arrive at the transfusion unit. Each $n^{\text {th }}$ pre-booked TFU patient was then scheduled to arrive at the $n^{\text {th }}$ time estimator and was prioritized over other patient groups.

\subsubsection{Low Wait Time Scenario}

In order to protect patients from waiting, patients should be scheduled late enough to ensure it is highly likely that the constrained resource can serve them right away. To model this scenario, we followed the same procedure as the one for the "high constraint utilization" scenario, except that the maximum time that the $\mathrm{n}^{\text {th }}$ pre-booked TFU patient was assigned to a treatment chair was used as the time estimator. Each $n^{\text {th }}$ pre-booked TFU patient was then scheduled to arrive at the $\mathrm{n}^{\text {th }}$ time estimator and was prioritized over other patient groups. 


\subsubsection{Drum-Buffer-Rope Scenario}

We explore a more balanced scenario in which patients are scheduled to arrive at TFU when there is a fair chance that there is a treatment chair available for them. To model this scenario, we followed the same procedure as the one for the "high constraint utilization" and "low wait time" scenarios, except that the $50^{\text {th }}$ percentile value of the time that the $\mathrm{n}^{\text {th }}$ pre-booked TFU patient was assigned to a treatment chair was used as the time estimator. Each $n^{\text {th }}$ pre-booked TFU patient was then scheduled to arrive at the $n^{\text {th }}$ time estimator and was prioritized over other patient groups.

\subsection{Results}

As expected, results from running the three scenarios (figure 3.2) demonstrated that the "drumbuffer-rope” scenario balances the trade-off between instances of delayed treatment and average patient wait times. With a mere 0.44 percent compromise (i.e. increase) in the instances of delayed treatment, the "drum-buffer-rope” scenario improved (i.e. decreased) average patient wait time by 34.15 percent when compared to the "high constraint utilization" scenario. On the other hand, with a 7.33 percent compromise (i.e. increase) in the average patient wait time, the “drum-buffer-rope” scenario improved (i.e. decreased) instances of delayed treatment by 14.25 percent when compared to the "low wait time" scenario

Please note that the average patient wait time reported in figure 3.2 is across all patient groups going through the transfusion unit (as opposed to only the scheduled ones), and that it is the pure 
wait time for treatment chairs at the transfusion unit (excluding possible previous waits for treatment product preparation either in the blood bank or pharmacy).

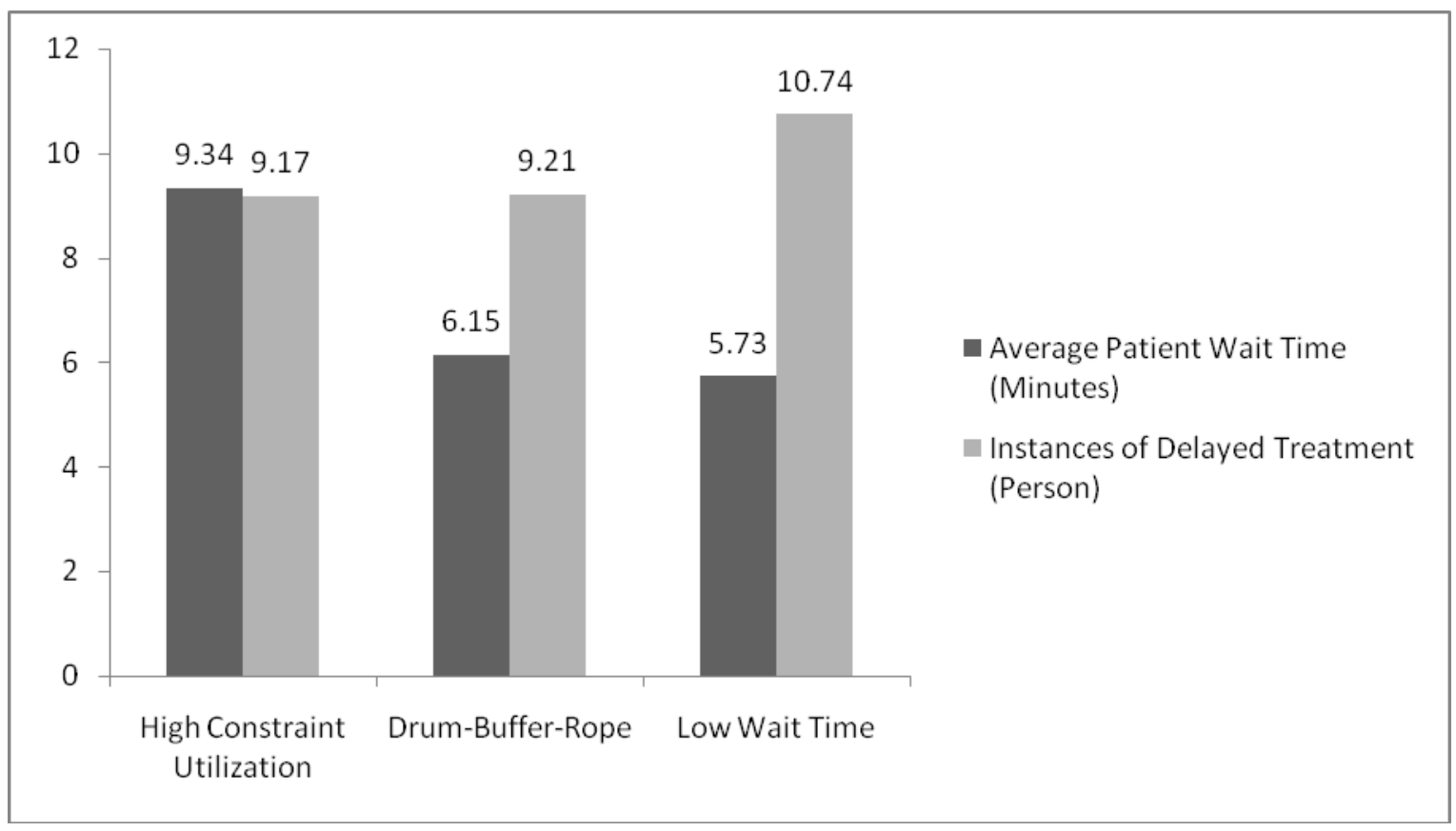

Figure 3.2: Simulated Performance Measures for the Three Scenarios

Figure 3.3 demonstrates the cumulative distribution of patient wait time (individual patient wait time, as opposed to the average patient wait time) at the transfusion unit for the three scenarios. It is observed that as we move away from the "high constraint utilization" to the "drum-bufferrope" and then towards the "low wait time”, the curve shifts to the left, meaning that a higher percentage of patients experience lower levels of wait times. The maximum wait time that patients had to experience in each of these scenarios, which is graphically demonstrated by where the curves end for each scenario, is also reduced as we move away from "high constraint utilization" to the "drum-buffer-rope” and then towards the "low wait time”. 


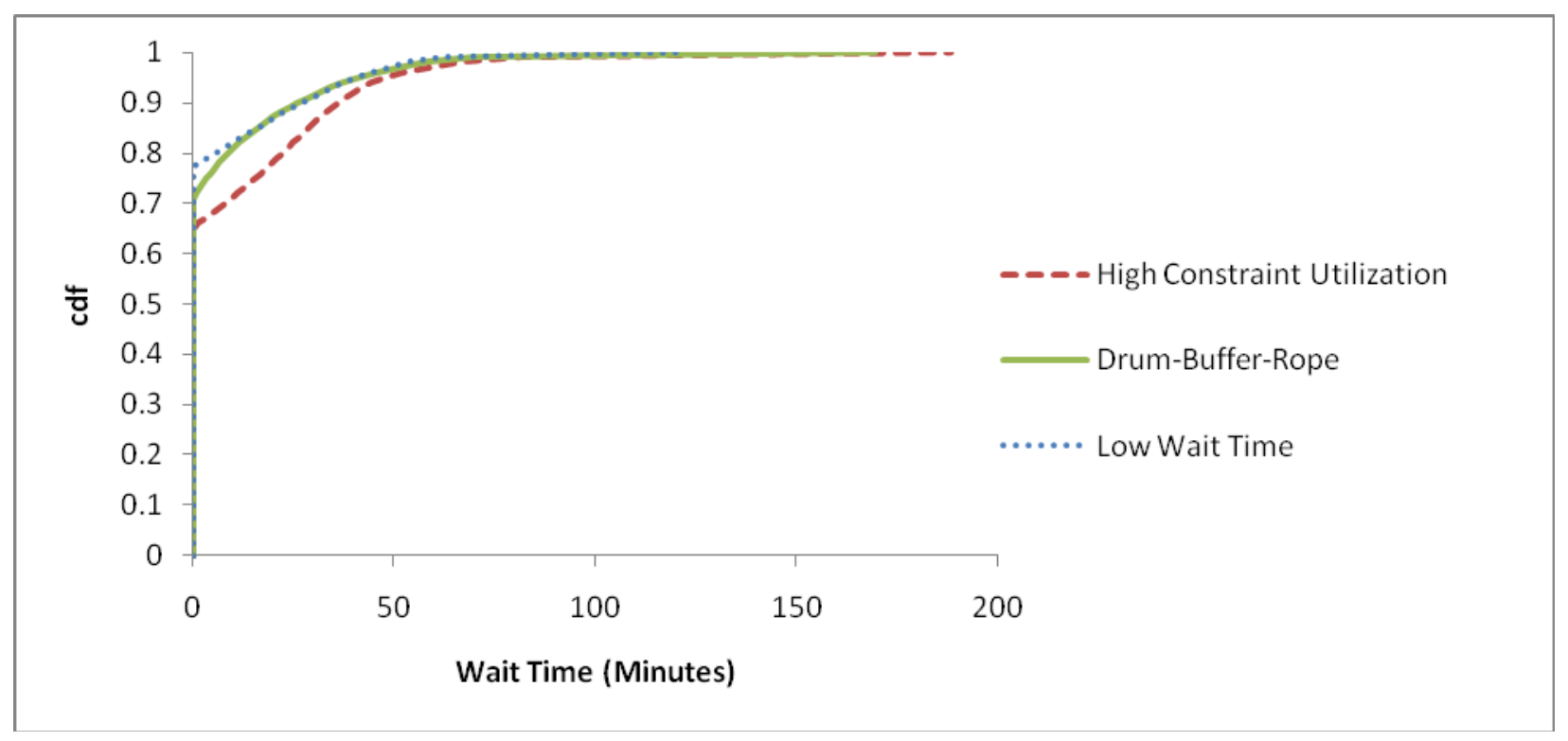

Figure 3.3: Cumulative Distribution of Patient Wait Time

\subsection{Discussion}

In developing the DBR strategy, we took the $50^{\text {th }}$ percentile of each $\mathrm{n}^{\text {th }}$ treatment chair availability times. We can choose different percentile values of the simulated $\mathrm{n}^{\text {th }}$ treatment chair availability times and use the simulation model to see the effects on the two performance measures of average patient wait time and instances of delayed treatment. Note that our "high constraint utilization" and "low wait time” scenarios are indeed using the zero and 100 percentile values of the $n^{\text {th }}$ treatment chair availability times respectively. Figure 3.4 shows the average patient wait time and instances of delayed treatment for the $10^{\text {th }}, 30^{\text {th }}, 50^{\text {th }}, 70^{\text {th }}$ and $90^{\text {th }}$ percentile value of each $\mathrm{n}^{\text {th }}$ treatment chair availability times, along with our original results for the "high constraint utilization scenario" and the "low wait time scenario" noted with percentile values of zero and 100 respectively. 


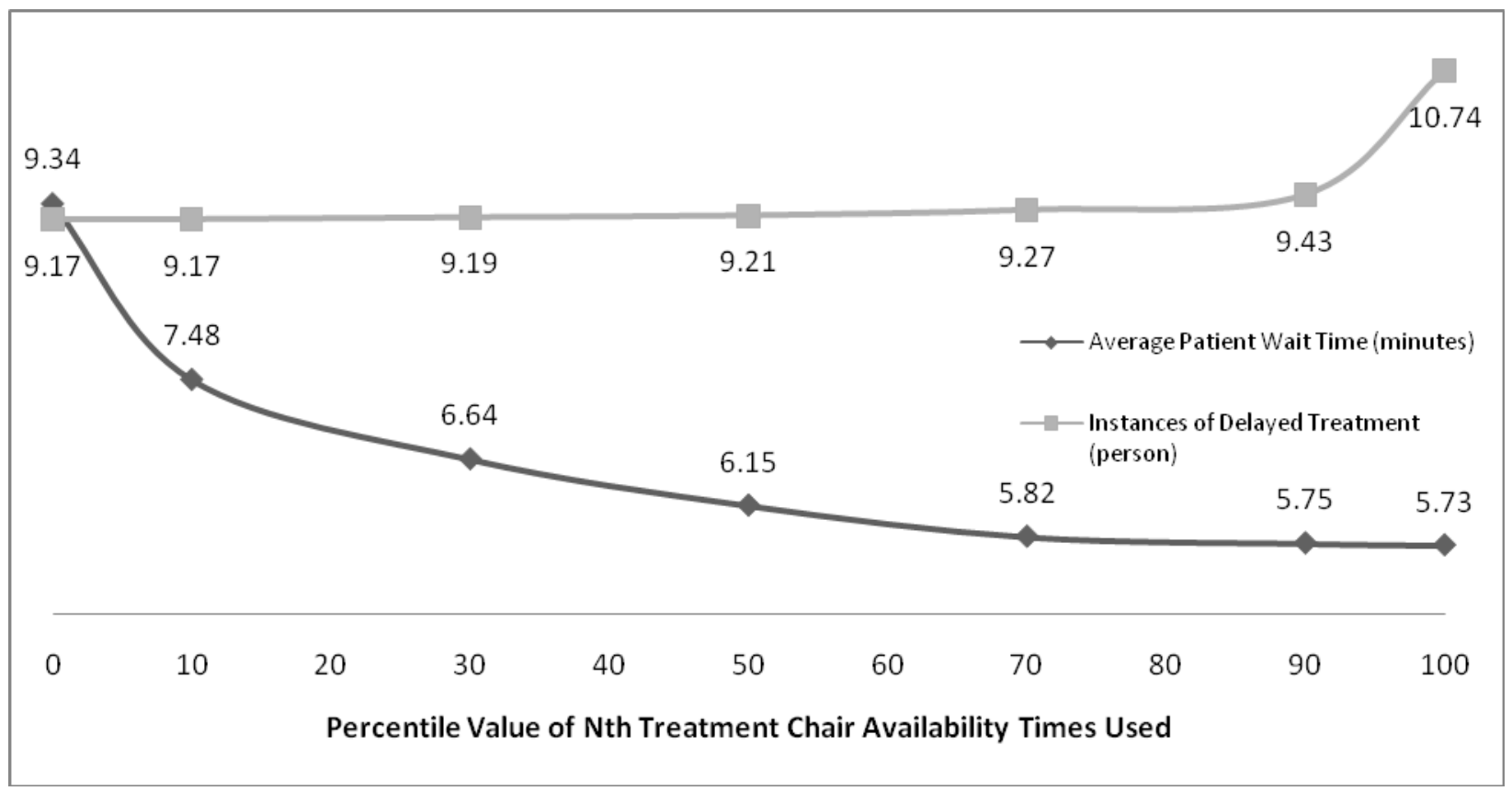

Figure 3.4: Performance Measure Values Resulting from Using Different Percentile Values of the Nth Treatment Chair Availability Times as Time Estimators in DBR

As expected, earlier scheduling of patients (i.e. using lower percentile values of $\mathrm{n}^{\text {th }}$ treatment chair availability times) generally results in higher wait times for patients, but a better protection of the constrain as demonstrated by lower instances of delayed treatment. What is notable in Figure 3.4 is that the slope of the "average patient wait time" curve is very sharp from zero percentile (i.e.: high constraint utilization scenario) to around the $70^{\text {th }}$ percentile, when, at the same time, the "instances of delayed treatment" curve is almost flat. So, there are potential large gains in reducing average patient wait time without significantly increasing instances of delayed treatment by increasing the percentile values to about 70 .

We would like to discuss here how the fact that our model might be underestimating the wait for morning clinic visit would affect this finding. If patients were to wait more than what they do now in the simulation model, they would, on average, arrive at the transfusion unit at a later time. This is equivalent to shifting our time estimates for the treatment chair availability times to 
later points of time. The later time estimates are equivalent to taking a higher percentile value of the treatment chair availability times in our current model. We have shown the finding that drum-buffer-rope compromises one performance measure in favor of the other to be true for a range of percentile values of the treatment chair availability times. This main finding would not change under a more real world representative model. However, the fact that in our model, one could gain a lot in wait time reduction without much compromise in instances of delayed treatment by increasing the percentile values taken to about $70^{\text {th }}$ percentile might or might not hold the same in a more real world representative model. There probably is the same trend of large gains in average patient wait time with little compromise in instances of delayed treatment up to a certain point, but the point might or might not be the $70^{\text {th }}$ percentile.

A more important point of discussion is that, while the simulation model can be used to quantify the level of compromise in one performance measure in favor of the other when choosing different percentile values of the simulated $n^{\text {th }}$ treatment chair availability times, the strategic question of what level of compromise is acceptable still remains unanswered.

To answer this question, we should look at the broader health system within which these services are provided. Why are these services funded? What is the goal of the health system? How do the average patient wait time and instances of delayed treatment affect the goal? What level of compromise would best serve the purpose of the system?

The services patients are receiving are publicly funded through taxes. Tax payers collectively fund the health system for a purpose, and the right level of compromise should be assessed with regard to its impact on the overall purpose of the system. Defining this overall goal of the system is no trivial task, as there are very different aspects to health systems. Fortunately, the theory of constraints provides a good framework to identify the system-wide goal and define global 
performance measures to assess it. Chapter four of this thesis takes a theory of constraints approach to derive an overall health system goal and define global performance measures that are capable of assessing this goal. With that, in chapter five, we provide a framework to assess the effects of low-level performance measures, such as average patient wait time and instances of delayed treatment in this case study, on global system-wide performance measures. Only when these effects have been adequately quantified can the right level of compromise in one low-level performance measure in favor of the other be determined. Before proceeding to the next chapter, however, we would like to discuss some of our observations about data availability and recommend strategies for improvement, as well as present some areas for future research.

\subsection{Data Issues and Recommendations}

Besides the direct findings of this research with regards to the effectiveness of a DBR scheduling policy in balancing the tradeoff between average patient wait time and instances of delayed treatment, there were lessons learned pertaining to data availability at the constrained resource stage that we would like to share.

Our experience confirmed Bolander and Taylor's (2000) finding that "TOC minimizes scheduling effort and data requirements by requiring significant data only for the bottleneck constraint". Once the bottleneck of the system has been identified, the DBR only requires detailed data with regards to the operation of the bottleneck (to be able to set the drum beat and buffer sizes). In the simulation, this was demonstrated by the need to only record the treatment chair availability times at the transfusion unit, but no other time log of the previous stages of the outpatient visit. This is both an advantage and disadvantage of the theory of constraints 
framework. On the positive side, if the data related to the operations of the bottleneck are available, it is possible to implement the scheduling system even if extensive data is not available at the non-constrained stages. On the negative side, it gets very important to have the right type and good caliber data available with regards to the operations of the constrained resource to be able to implement DBR.

What we found was that more often than not, the infrastructure to obtain these data points was either available or easy to develop, but for various reasons the staff were not motivated to record the data. Especially at the constrained resource stage, staff are usually extremely busy making sure that the limited resource is used to the best extent possible and see such record keepings as time taken away from immediate patient care operations. We believe that it is important to educate the staff on the positive effects of correctly recording such operational data by explaining how this task would ensure better planning of constrained resource utilization and eventually a better patient care through a process of ongoing improvement. Patients could be asked to record the data as well, for instance by using sign-up sheets to record the arrival times and distributing patient self-track forms. To ensure the continuity of recording such data, the management could ask for operational reports to be included as part of the more routine departmental patient care reports. As noted before, and as an advantage of the theory of constraints framework, these rigorous record keeping efforts are only required at the constrained resource stages and not at each and every stage of the process.

In summary, to successfully implement the theory of constraints framework, there is a need for strong leadership and participation of various stakeholders and most importantly, as Young et al. (2004) mention, the perspective that "we should not expect to invent systems that work perfectly immediately but rather that a process of gradual improvement should be designed into them, with 
all stakeholders participating in the improvement process. Most radically, this might include patients themselves”.

\subsection{Future Research}

This research examined a simple DBR based scheduling policy. Future research can look into more complicated policies, for instance those based on an intelligent based sequencing of prebooked TFU patients. As an example, it might make a difference on the performance measures of average patient wait time and instances of delayed treatment if pre-booked TFU patients are scheduled from the longest to shortest expected treatment time, or vice versa. For the sake of our simple DBR based scheduling policy, we assume a random sequence (that was kept the same for the three scenarios, as part of the common random numbers approach used in the comparison of the scenarios). Furthermore, user friendly interfaced models could be developed and transferred to the hospital to use the simulation model as an ongoing planning tool in their constantly changing environment.

Moreover, this research had assumed that improvement initiatives to reduce the processing times as much as possible, like the ones discussed by Kershaw (2000), have been accomplished before implementing the DBR based scheduling policy. Future research could look into various types of such improvement initiatives to exploit the constraint, including but not limited to lean thinking (Womack and Jones, 2003) and the complete kit concept (Leshno and Ronen, 2001). 


\subsection{Summary and Conclusions}

This research was motivated by a common perception that even though the quality of clinical care received by outpatients in the cancer facility under study was excellent, there was room for improving the overall experience. To tackle the various uncertainties in a typical outpatient visit that hinder proper advance planning, we looked into the theory of constraints framework and specifically its drum-buffer-rope technique. Using a discrete event simulation model populated with historical data, we compared the DBR based scheduling policy against "high constraint utilization" and "low wait time" scenarios. The DBR based scheduling policy proved to be an effective mechanism in balancing the inherent tradeoff between the two performance measures of instances of delayed treatment and average patient wait time. However, the level of compromise in the level of one performance measure to achieve an enhanced level in the other is a strategic question that requires further research in relating the performance measures in this chapter to the system-wide TOC performance measures for publicly funded health care systems developed in the next chapter. We shared some lessons learned and made recommendations with regards to data keeping practices at the constrained resource stage. Finally, we proposed an array

of future research including a more intelligent sequencing of pre-booked patients at the constrained stage. 


\section{TOC’s Performance Measures for Publicly Funded Health Systems}

\subsection{Abstract}

Originally developed in the context of publicly traded for-profit companies, theory of constraints (TOC) improves system performance through leveraging the constraint(s). While the theory seems to be a natural fit for the resource-constrained publicly funded health systems, there is a lack of literature addressing the required customizations especially with regards to defining the goal and performance measures. This chapter develops a system dynamics representation of the classical TOC's system-wide goal and performance measures for publicly traded for-profit companies, which forms the basis for developing a similar model for publicly funded health systems. The model is then expanded to include some of the factors that affect system performance, providing a framework to apply TOC's process of ongoing improvement in publicly funded health systems. Future research is required to more accurately define the factors

affecting system performance and populate the model with evidence-based estimates for various parameters in order to use the model to guide TOC's process of ongoing improvement. 


\subsection{Introduction}

Theory of constraints (TOC), first developed in manufacturing by Eliyahu Goldratt (Goldratt and Cox, 1984), views systems and processes as series of dependent events. Like the fact that a chain is only as strong as its weakest link, all systems have at least one and at most a few constraints that limit system performance (Womack and Flowers, 1999). TOC emphasizes that improving constraint's performance directly results in enhancing total system performance (Breen et. al., 2002). Thus, TOC gives insight on process improvement efforts.

TOC's process of ongoing improvement has five steps. First, system constraints are identified. Second, they are exploited by making physical constraints as effective as possible and eliminating policy constraints (replacing them with policies allowing exploitation of the constraints). The third step is to subordinate everything to the constraint. At this stage nonconstraint resources are operated in a way to support the plan to exploit the constraint. If the system performance is not enhanced to the desired extent, in the fourth stage the constraint is elevated by increasing its capacity (potentially a costly stage and therefore a strategic decision). Recognizing the fact that the system constraint has probably shifted, the final stage is to overcome inertia and start over from the first step to identify the new system constraint. (Rahman, 1998)

A prerequisite to implementing TOC's process of ongoing improvement is measuring the performance of different stages of the system with regards to the goal. Therefore, a goal along with appropriate performance measures is needed. The classical TOC literature has fully discussed the goal and performance measures for publicly traded for-profit companies (Goldratt, 1990). But the equivalent literature for publicly funded health systems is scarce, and does not 
cover all of the performance measures. The purpose of this chapter is to fill the gap in the literature by finding the equivalents of TOC's classical goal and performance measures for publicly funded health systems and build a conceptual framework for implementing TOC's process of ongoing improvement in this setting.

To set the context, a system dynamics (SD) representation of the goal and performance measures for publicly traded for-profit companies is presented in the next section. This representation forms the foundation to construct a similar model, capable of defining the relationships between the suggested goal and performance measures, for publicly funded health systems. The model is then expanded to include some of the factors that affect system performance, providing a framework to apply TOC’s process of ongoing improvement in publicly funded health systems. For brevity reasons, we use the term "health system” in lieu of "publicly funded health system” in the rest of this chapter.

\subsection{An SD Model of TOC’s Classical Goal and Performance Measures}

TOC defines the goal of the system from the perspective of its owners. In classical TOC literature dealing with publicly traded for-profit companies, shareholders are the owners and they define the goal as making money, both now and in the future. The performance of the company is measured with regards to the goal and through the global performance measures of net profit, return on investment, and cash flow. Moreover, a set of operational performance measures, namely throughput, inventory, and operating expenses, are defined that enable company management to assess the effects of local actions on global performance measures. In-depth explanations of these performance measures are discussed by Goldratt (1990). 
There is a hierarchy amongst the global performance measures. At the bottom is the survival performance measure of cash flow. One would not invest in a company expected to go bankrupt. At the next level, the more the company makes money (the absolute measure of net profit), the more appealing it is for investment. But the ultimate performance measure is the relative performance measure of return on investment: how much money is made relative to the amount invested.

To shed more light on the descriptive explanations of these measures in the literature and demonstrate the relationships between TOC's classical goal and performance measures, we developed a system dynamics (SD) stock-and-flow diagram as depicted in figure 4.1. Throughout our descriptions of the model below, the terms in italics refer to specific model variables in figure 4.1. The fundamentals of system dynamics are well described by Sterman (2000).

There are two stock variables: Total Cash and Inventory. Inventory is all the money the system has invested in purchasing things it intends to sell. While at first sight this appears to only include the inventory of materials, it also encompasses any other facility or equipment that the company possesses. The company can and will sell these possessions when they are not required anymore. However, it should be noted that the value of inventory is calculated only based on the purchase price. The concept of "value added" used in cost accounting is not practiced in TOC, as it could mislead the management to take local actions that do not improve the overall performance. (Goldratt, 1990)

Each time a new investment is made; the Investment flow deducts the amount from the Total Cash stock and adds it to the Inventory stock. Inventory is depreciated via two flows: Investment Gained Back and Investment Waste or Depreciation. The Investment Gained Back flow is the 
rate at which part of the investment is gained back through sales (e.g. sales of material, equipments, or facilities). As such, the Investment Gained Back flows back to the Total Cash stock. In calculating this rate, only the purchase price of the investment being sold is considered. The potential extra money generated (above the purchase value) is the throughput in TOC language: "the rate at which the company generates money through sales" (Goldratt, 1990). Throughput is another input to the Total Cash stock.

Inventory can also depreciate through waste of investments (e.g. scrapped goods), or depreciation (loss of value) of equipment and facilities over time. This is shown by the Investment Waste or Depreciation flow. As the money wasted or depreciated is not gained back through sales, this rate does not flow back to the Total Cash stock.

Like all stock variables in system dynamics, the value of the Total Cash stock at each time $\mathrm{T}$ is formulated as the accumulation of its input minus its output flows, plus its initial value:

$\operatorname{Total} \operatorname{Cash}(T)=\int_{0}^{T}($ Throughput $(\mathrm{t})+$ Investment Gained Back(t) - Investment $(\mathrm{t})-$ Overhead Cost $(\mathrm{t})) \mathrm{dt}+\operatorname{Total}$ Cash $(0)$

Similarly:

$\operatorname{Inventory}(T)=\int_{0}^{T}(\operatorname{Investment}(\mathrm{t})$ - Investment Gained Back(t) - Investment Waste or Depreciation $(\mathrm{t})) \mathrm{dt}+\operatorname{Inventory}(0)$

The Total Cash stock serves a double role: on the one hand, it is all the money the company has at any time: it is the goal units available at present. In other words, the goal is to increase the Total Cash stock over time. On the other hand, it should never hit zero or the company will go bankrupt. In TOC’s language, it is the survival performance measure of cash flow. 
The Total Cash stock is increased by Investment Gained Back and Throughput Flows. It is decreased by Investment and Overhead Cost flows. Overhead cost is the rate of spending money to operate the company (e.g.: rent, salaries, and inventory carrying charges). These expenditures are not gained back through sales, and as such are not considered investments.

The exact formulations of the various flow rates depend on the special circumstances of the company. For instance, the Overhead Cost flow might be some fixed value per year plus a function of the level of the inventory. Investment Wasted or Depreciation flow could be a percentage of the inventory that is scrapped or depreciated per year. Investment flow could include the purchase of material and capital investment and might be formulated based on the volume, purchase price, and frequency of these purchases.

The above variables basically model the flow of cash within the company. Since the stock variables are expressed in dollars, all the flow variables are expressed in dollars per some period of time, for instance dollars per year.

The other performance measures, modeled as auxiliary variables, are formulated as follows: (the terms in brackets show the units of measurement)

Operating Expense $\left[\frac{\$}{\text { Year }}\right]=$ Overhead Cost + Investment Waste or Depreciation

Net profit $\left[\frac{\$}{\text { Year }}\right]=$ Throughput - Operating expense

Return on investment $\left[\frac{1}{\text { Year }}\right]=\frac{\text { Net profit }}{\text { Inventory }}$ 


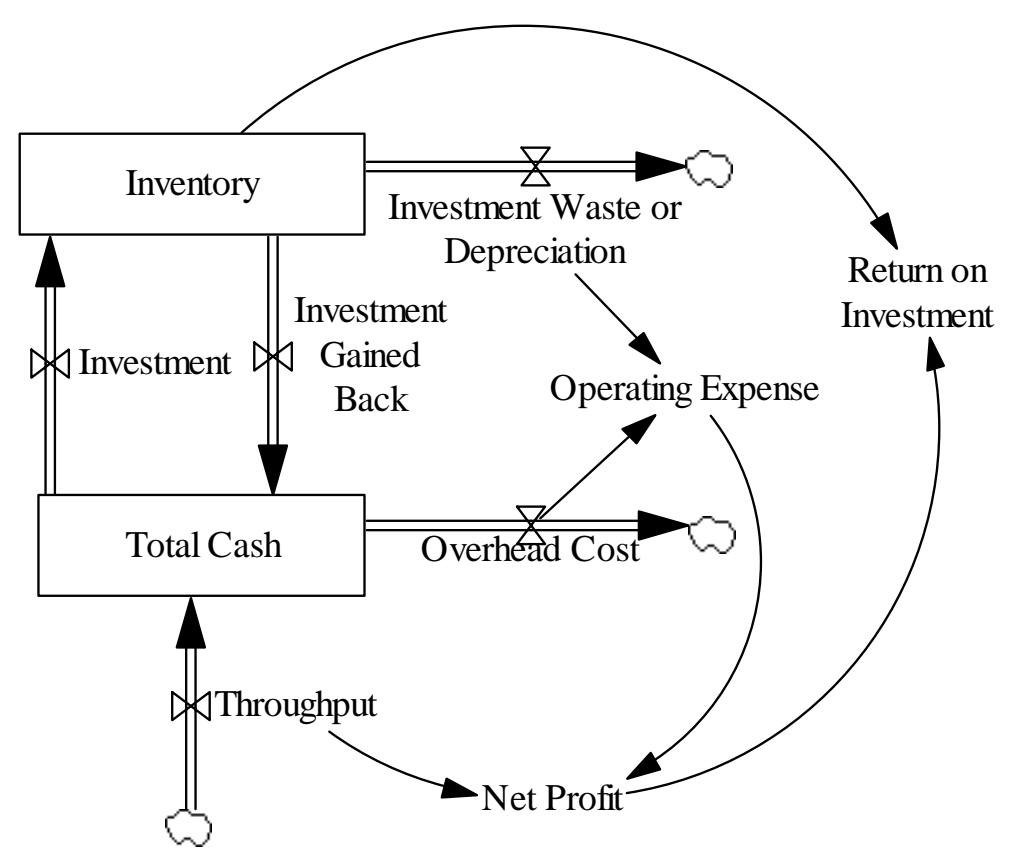

Figure 4.1: TOC’s Classic Goal and Performance Measures

A final point to discuss here is discounting the value of money over time as commonly practiced in engineering economics. Auxiliary variables that represent the equivalents of any of the monetary variables (those measured in $\$$ or $\$ / Y e a r)$ at time zero can be easily added to the model and formulated by using the continuously compound interest rate formula: $P=\frac{F}{e^{r N}}$ (Riggs, 1996), where $\mathrm{r}$ is the interest rate per period of time and $\mathrm{N}$ is the number of time periods between P (present value) and F (future value). To keep the diagram simple, we have not added these auxiliary variables to the model.

Having successfully modeled TOC's definition of the goal and performance measures for publicly traded for-profit companies, we now move forward to define the equivalent goal and the performance measures for health systems. 


\subsection{Defining the Goal for Health Systems}

The TOC literature has suggested a few goal statements for the health industry, including "to maximize life expectancy and quality of life at an acceptable cost to society” (Hunink, 2001), “to maximize the units of health" (Breen et. al., 2002), "providing safe care while thinking of money only as an operating expense - something to be reduced" (Mcnutt and Odwazny, 2004), "to treat more patients both now and in the future" (Wright and King, 2006), to "maximize quality medical services provided to its customers, subject to budgetary constraints” (Ronen et. al., 2006), and "to make more health, today as well as in the future" (Schaefers et. al., 2007).

In developing the goal, we follow the same logic in deriving the goal for publicly traded forprofit companies in that the goal should be defined by the owners of the system. We see tax payers as the true owners because similar to shareholders investing in publicly traded for-profit companies, tax payers are investing in health systems.

We propose that tax payers fund health services so that the society enjoys longer and higherquality lives in general. Therefore, we define the goal as: to increase the quality and quantity of lives both now and in the future. This goal statement leads us to choose the quality adjusted lifeyears (QALY) as the unit of measurement for the goal. The advantage of using QALY as the unit of measurement is that it simultaneously captures gains from reduced morbidity (quality gain) and reduced mortality (quantity gain), and combines these two into a single measure (Drummond, 1987). In fact, in discussing a different topic (the accuracy of QALY as a utility), Drummond (1987) also says: "The view is that the QALY is a good basic definition of what we are trying to achieve in health care, and maximizing QALYs is quite an appropriate goal”. 
We differ in the goal statement with those suggesting treating more patients both now and in the future (Wright and King, 2006). We think the society does not fund the system to maximize the number of patients treated, as, for instance, the society might prefer that there are no patients in the first place to be treated (i.e. favor prevention over treatment where possible and economically feasible). Our goal statement is similar to the other goal statements mentioned above. However, we believe that our expression of the goal allows for a more accurate development of TOC's operational and global performance measures for health systems, as described in the following section.

\subsection{An SD Model of TOC’s Performance Measures for Health Systems}

If there are different, but not totally disagreeing, statements of the goal for health systems, the issue just gets more complicated when defining the performance measures. Except for Schaefers et. al. (2007), none of the papers in the literature try to define all the performance measures in a systematic way and relate them back to the goal. Schaefers et. al. (2007) formulate the goal with some units of life time expectancy multiplied by an index of life quality, and try to link the performance measures to this goal. Their fundamental idea in formulating the performance measures has some similarities to ours, but it falls short in showing a comprehensive relationship between all the performance measures and the goal, especially when it comes to operating expenses.

We use a system dynamics framework to define and formulate the relationships among the goal and the various performance measures. Our goal is to find the equivalents of the variables in figure 4.1 for health systems. We accomplish this goal in two steps. First, we model the goal and 
the performance measures from the perspective of one individual interacting with the health system by investing or spending goal units (QALYs) with the expectation to gain more goal units (QALYs) in the future, as shown in figure 4.2. Next, we scale up the model to incorporate all the individuals in the society and formulate the goal and performance measures at the health system level (figure 4.3).

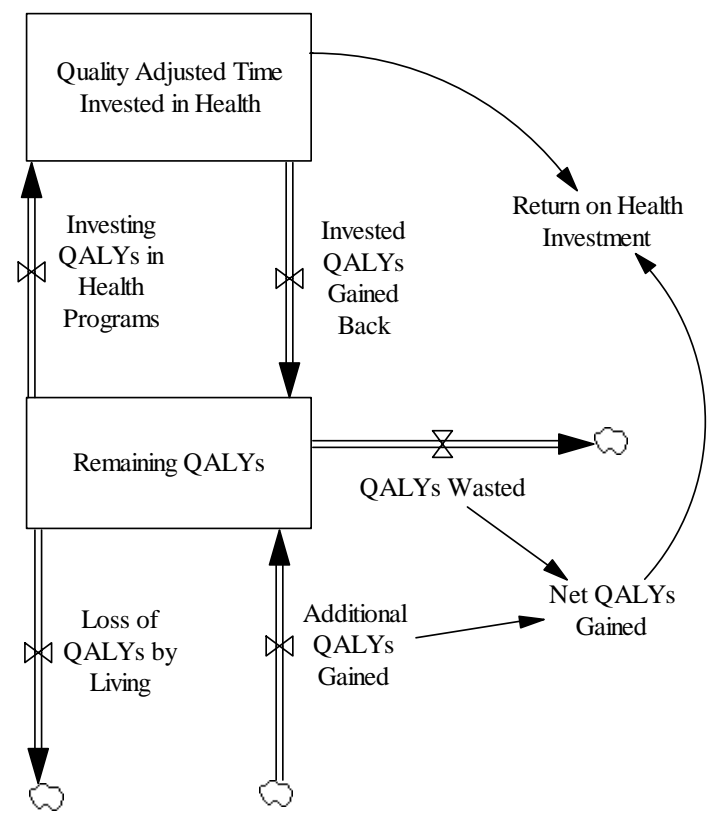

Figure 4.2: Health System's Goal \& Performance Measures - Individual's Perspective

Figure 4.2 displays the equivalents of the variables in figure 4.1 for an individual interacting with the health system. The individual chooses to take advantage of the health system for one purpose: to increase her Remaining QALYs or the QALYs ahead of her at each point of time. Although the value of the Remaining $Q A L Y$ s at any point of time is unknown to any individual, this value does exist at any point of time since, at least in theory, it can be determined retrospectively at the time of death. The Remaining QALYs stock is the equivalent to the Total 
Cash stock in figure 4.1. But there is a slight difference between the two. Unlike the Total Cash stock that only diminishes when there is some activity (investment or operating expenses); the Remaining QALYs diminishes even if there is no activity. That is, even if one does not interact with the health system at all, her Remaining QALYs stock diminishes as time passes (and eventually hits zero at the time of death). So, there is a need for a natural diminishing of QALYs in the model. This is done through the Loss of QALYs by Living flow. This flow simply represent living life: losing one QALY during a year that one is in perfect health, losing half a QALY in another year when one is half as healthy, etc. Also, it is possible that one also gains some QALYs, for instance through exercising. In this case, the rate of Loss of QALYs by Living is adjusted to include the rate of QALYs gained; possibly turning the rate of Loss of QALYs by Living negative if the rate of gaining QALYs is higher than the rate of losing them over a certain period of time. Please note that the Loss of QALYs by Living flow only captures gains and losses (and the drain of Remaining QALYs stock at death) of the Remaining QALYs stock due to reasons other than interacting with the health system. The other flow variables, as described below, model the interactions with the health system.

The rest of the model is almost an analogy to figure 4.1. The individual might choose to participate in various health programs with the expectation that this participation will increase her Remaining QALYs stock in the future. We use the term health program very widely here, including any activity or intervention offered by the health system that is expected to increase the quality and/or length of life such as health education and prevention, treatment, rehabilitation, and even palliative care (intended to solely increase the quality of life).

Since participating in health programs is an investment in health, we model it with a flow similar to the Investment flow in figure 4.1 and call it Investing QALYs in Health Programs. It is defined 
as the rate at which the individual invests QALYs in health programs. It should be noted that the Losing QALYs by Living flow is adjusted to only include the QALYs lost due to reasons other than participating in health programs. As such, if someone in perfect health invests one hour a day in health programs (i.e. invests 1/24 QALYs per year), the Losing QALYs by Living rate would be 23/24 QALYs per year, since 1/24 QALYs per year is accounted for in the Investing QALYs in Health Programs flow. In other words, the perfectly healthy individual is still losing one QALY per year in total, but $1 / 24^{\text {th }}$ of that is a different kind of losing: one that is expected to increase the Remaining QALYs stock in the future and as such is viewed as an investment.

The equivalent for the Inventory stock in figure 4.1 is what we call Quality Adjusted Time Invested in Health stock in figure 4.2. These are the total QALYs the individual has lost (in fact invested) due to participating in health programs for which the health benefits have not been realized yet. The time to realize health benefits varies greatly from one health program to another. It could be almost immediate (such as a laser eye surgery), or quite lengthy (such as speech therapy programs with benefits that are gained gradually over a long period of time). Once the benefits are gained, they increase the Remaining QALYs stock. We have broken down the gain into two flows: Invested QALYs Gained Back which is part of the gain that equals the total investment for the specific health program whose benefit is being realized, and Additional QALYs Gained, equivalent to the Throughput flow in figure 4.1, that captures the difference between the gain and the total investment for the same health program. This difference is usually expected to be positive, or else the individual will not participate in the health program. However, it is possible that the health program does more harm than good. For instance, a chemotherapy regimen may prove to be ineffective and returns less QALYs than invested in the program or no QALYs at all. Another example is a preventive or treatment intervention that 
diminishes (or possibly totally drains) the Remaining QALYs stock due to an accident or medical error. In situations like these, the Additional QALYs Gained flow is negative.

An interesting point here is that figure 4.2 does not have an equivalent to Investment Waste or Depreciation flow in figure 4.1. The reason is that unlike the Inventory stock in figure 4.1, which existed in the real world and could be wasted or depreciated, the Quality Adjusted Time Invested in Health stock is the QALYs already lost (albeit invested) that do not exist anymore and cannot be wasted or depreciated. We only keep track of them since they are expected to return some QALY gains at some point in the future (and if they don't, they will result in a negative Additional QALYs Gained flow, as explained above).

The final flow in figure 4.2, QALYs Wasted, is the rate at which QALYs are wasted when one participates in a health program. Examples include time spent to commute to the health program location or to wait around during the various stages of participating in a health program. These are wasted QALYs (as opposed to invested QALYs), since no health benefit is expected as a result of losing them. However, given the current circumstances, including a shortage of capacity or possible inefficiencies in the system, the individual has to lose them in order to be able to participate in health programs. In other words, this flow is equivalent to the Overhead Cost flow in figure 4.1. Since figure 4.2 does not have an equivalent to Investment Waste or Depreciation flow of figure 4.1, QALYs Wasted is the equivalent to the Operating Expense of figure 4.1 as well. Similar to the case of Investing QALYs in Health Programs flow, the Loss of QALYs by Living flow has to be adjusted to exclude those QALYs accounted for in the QALYs Wasted flow.

The two stock variables of the model in figure 4.2 are measured in units of QALY and are formulated as the accumulations of their input flows minus their output flows over time, plus 
their initial values at time zero. All the flow variables of the model are measured in QALY/Year. As with the case in figure 4.1, the exact formulations of the flow variables depend on the special circumstances.

As for the performance measures, similar to the Total Cash stock in figure 4.1, the Remaining QALYs stock serves the double role of the current level of goal units, as well as the survival performance measure, equivalent to cash flow in TOC language. Similar to the company going bankrupt if the Total Cash stock hits zero, the individual is dead if the Remaining QALYs stock reaches zero.

The last two global measures are formulated as follows (the terms in brackets are the units of measurement):

Net QALYs Gained [ $\left.\frac{Q A L Y}{\text { Year }}\right]=$ Additional QALYs Gained - QALYs Wasted

Return on Health Investment $\left[\frac{1}{\text { Year }}\right]=\frac{\text { Net QALY }}{\text { Quality Adjusted Time Invested in Health }}$

A final note here is discounting future QALYs, as if a QALYs gained (or spent) today is worth more than one gained (or spent) later. Unlike in engineering economy where there is a general consensus around the need to discount the value of money over time, health economists are divided on their opinion around discounting health statuses. An interesting example of arguments against discounting is that "discounting years of life gained in the future gives less weight to future generations in favor of the present one" (Drummond, 1987). There are of course arguments in favor of discounting as well, including "simple numerical examples showing that leaving effects undiscounted while discounting costs, or discounting costs and effects at different 
rates, can lead to inconsistencies in reasoning” (Drummond, 1987). While the debate is definitely in the realm of health economics and not the subject of this research, we suffice to say that should a decision to discount is made, the model can be easily modified to incorporate auxiliary variables representing the equivalents of the model variables at time zero, using the same continuous compound discount formula mentioned in section 4.3. To keep the diagram simple, we have not included these auxiliary variables.

To elevate the model at the societal level, all we need to do is to aggregate the values of the various flow variables across the whole population. With two exceptions, Net QALYs Gained due to Immigration flow and Total QALYs Gained through Birth flow, each of the flow variables, as shown in figure 4.3, are the summation of the equivalent flow variable in figure 4.2 for all the individuals in the society. For instance, the Total QALYs Wasted flow is the summation of all the QALYs Wasted flow values of figure 4.2 for all the individuals in the society.

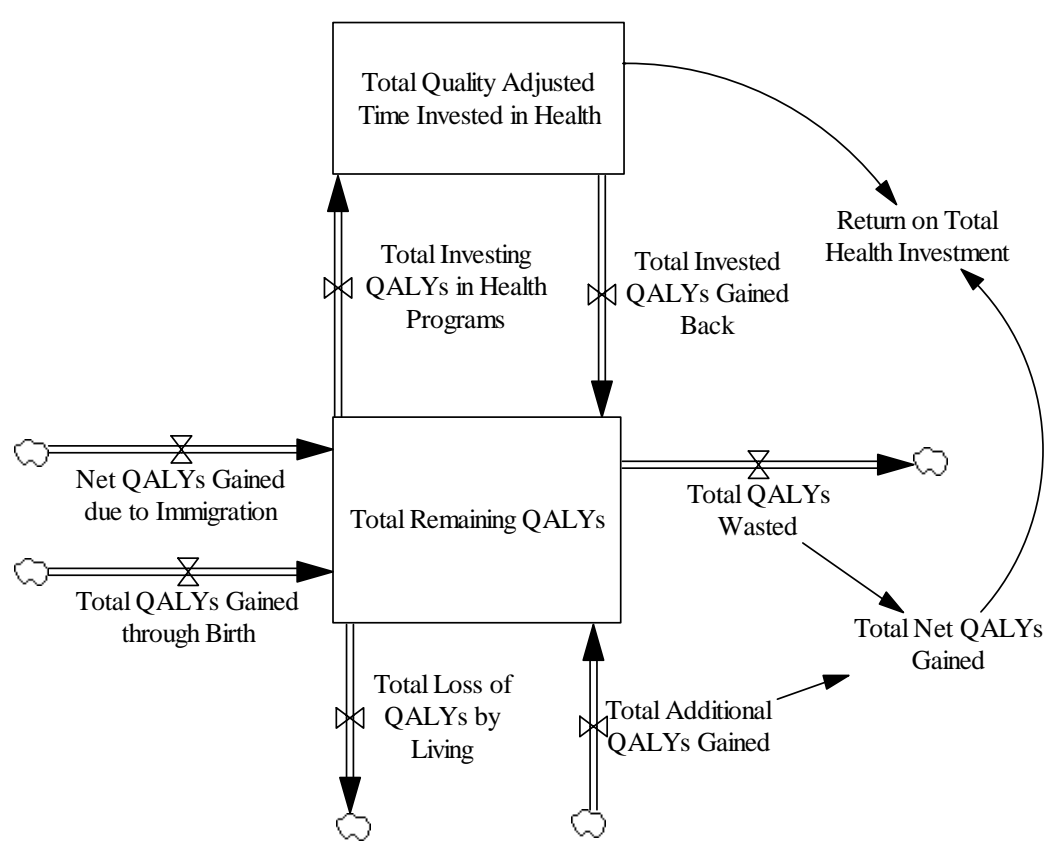

Figure 4.3: TOC’s Goal and Performance Measures for Health Systems 
The Net QALYs Gained due to Immigration flow variable captures the rate of change in the Total Remaining QALYs stock as a result of immigration. The rate is positive if there are more QALYs added to the Total Remaining QALYs due to immigration into the society than the QALYs lost due to emigration from the society and negative otherwise. The Total QALYs Gained through Birth flow captures the rate of increase in the Total Remaining QALYs stock through the birth of new babies. Any QALYs invested and wasted to provide health programs to the new immigrants and the new born babies is still captured with the Total Investing QALYs in Health Programs and Total QALYs Wasted flows respectively, and the gains are still modeled with the Total Invested QALYs Gained Back and Total Additional QALYs Gained flows. The latter might be a negative flow if the health program does more harm than good, for instance if the baby dies due to an adverse event. Similar to the individual level model, deaths due to reasons other than the performance of the health system (e.g. accidents) are modeled through the Total Loss of QALYS by Living flow.

The stock variables are still the accumulation of their input minus their output flows (as defined above) over time, plus their initial value at time zero. The initial value at time zero is determined by summing the values of the corresponding stock variable of all the individuals in the society.

The auxiliary variables are defined in the same way they were defined for the individual model (figure 4.2), except that they are based on the stock and flow variables in figure 4.3:

Total Net QALYs Gained = Total Additional QALYs Gained - Total QALYs Wasted

Return on Total Health Investment $=\frac{\text { Total Net QALY }}{\text { Total Quality Adjusted Time Invested in Health }}$ 
Similar to the case of publicly traded for-profit companies, there is a hierarchy amongst the global performance measures. At the bottom is the survival performance measure of Total Remaining QALYs. Individuals would not participate in health programs expected to empty this stock variable or in other words kill them. At the next level, the more the health program creates QALYs (the absolute measure of Net QALYs Gained), the more appealing it is to participate in. But the ultimate performance measure is the relative performance measure of Return on Health Investment or how many QALYs is created relative to the amount invested.

Finally, the QALYs can be discounted by adding the auxiliary variables representing the equivalents of the model variables at time zero and formulated by the same continuous compound discount formula mentioned in section 4.3. To keep the diagram simple, we have not included these auxiliary variables.

\subsection{A Framework for Applying TOC’s Process of Ongoing Improvement in Health Systems}

Having completed the prerequisite steps of defining the goal and performance measures, we should now be able to articulate TOC's process of ongoing improvement. The first step is to identify the bottleneck: What limits the performance of the system (as measured by the defined performance measures) the most. The goal and performance measures are fundamentally defined by the various flow variables of the model in figure 4.3. However, since we have not formulated the flow variables, it is not possible to determine what is most limiting the goal through the flow variables. 
While the factors affecting flow variables differ from system to system and as such developing accurate formulations applicable to any health system is not possible without extensive further research, it is our intention to present some of the likely factors and their possible effects on the flow variables and use them to discuss, at a conceptual level and through an example, how a more accurate and complete model can potentially guide TOC's process of ongoing improvement.

Figure 4.4 is a snapshot of the model with the additional factors. Please note that variables shown in $<$ and $>$ signs are the repetition of the variable with the same name and not between $<$ and $>$ signs presented elsewhere in the model. Moreover, since we are not formulating the flow variables here, only the direction of the relationship is shown in figure 4.4. A positive sign on the arrow connecting A to B means that, all else equal, if A increases (decreases), then B "increases (decreases) above (below) what it would have been” (Sterman, 2000). A negative sign means that, all else equal, if A increases (decreases), then B "decreases (increases) below (above) what it would have been” (Sterman, 2000).

Given that in publicly funded health systems there is usually more demand than capacity, Total Investing QALYs in Health Programs flow is mostly affected by effective capacity of the health system. We use the adjective "effective" to identify sections of the capacity that are not wasted. Moreover, "capacity” is an aggregate term encompassing both physical capacity (facilities, equipments, devices, etc.) and health human resources (physicians, nurses, psychiatrists, public health professionals, etc.). 


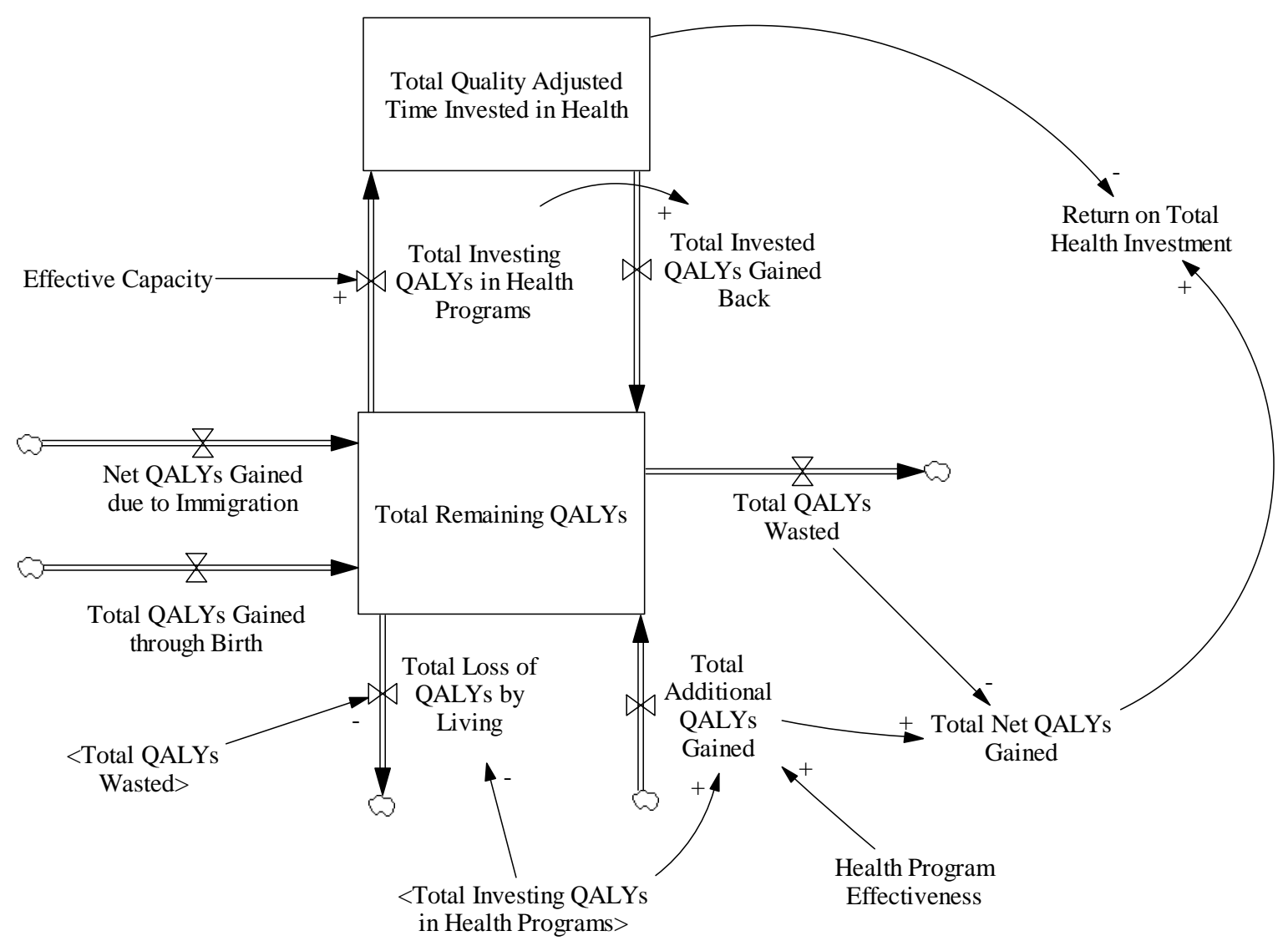

Figure 4.4: A conceptual model to guide TOC's improvement process in health systems

The values of the Total Invested QALYs Gained Back flow and the Total Additional QALYs Gained flow depend on the Total Investing QALYs in Health flow, since QALYs need to be invested in health programs before they can be gained back or additional (albeit sometimes negative) ones achieved. The value of Total Invested QALYs Gained Back is positively affected by Total Investing QALYs in Health flow, as the former is the rate at which the latter is gained back (after some benefit realization period).

The effect of the Total Investing QALYs in Health Programs flow on the Total Additional QALYs Gained can be either positive or negative and depends on the value of Health Program 
Effectiveness. Health Program Effectiveness captures the clinical appropriateness and quality aspects of the health service provided. Highly effective health programs return high levels of QALYs for every QALY invested, whereas less effective health programs return little in return, or even take away QALYs (when the health program does more harm than good). It should be noted that a health service program might be highly effective for certain populations, and less effective for others.

With a positive Health Program Effectiveness, the more the Total Investing QALYs in Health Programs flow, the more the Total Additional QALYs Gained flow will be (after the benefit realization period). If the Health Program Effectiveness is negative (i.e. health program doing more harm than good), then the value of the Total Additional QALYs Gained flow will be negative and the more the Total Investing QALYs in Health Programs flow, the less (or indeed the more negative) the value of the Total Additional QALYs Gained Flow will be (after the benefit realization period).

Total Additional QALYs Gained Flow is also positively affected by an improvement in Health Program Effectiveness, which can be improved through health research (both fundamental and clinical research), technological innovations resulting in more effective medical devices and technologies, and quality initiatives such as those aimed at reducing adverse events or enforcing best practice adoptions.

Though not obvious at first sight, the Total Loss of QALYs by Living flow is affected by the health system as well: the more QALYs are spent for health programs, either invested through Total Investing QALYs in Health Programs or wasted through Total QALYs Wasted, the less QALYs will be available to be spent for other purposes of life; in other words, the less will be the value of the Total Loss of QALYs by Living flow. 
The Net QALYs Gain due to Immigration flow is treated as exogenous and not analyzed here. Besides factors beyond health systems affecting the rate of immigration (e.g. political environment, economy, security, etc.), there might be factors related to health systems that influence this flow. But it is not the purpose of our model to explore these factors, nor do we intent to explore the factors affecting Total QALYs Gained through Birth flow.

Now that we have briefly introduced some of the factors affecting the flow variables (which themselves affect the performance measures), we would like to discuss how the model can be used to guide TOC's process of ongoing improvement with an example. First the bottleneck of the system has to be identified. Ideally, more accurate formulations of the relationships between the various factors and the performance measures have been developed and the initial values of the stock variables have been estimated based on population level estimates (for instance by looking into measures of population life expectancies and expected quality of lives, as well as aggregate estimates of the Total Remaining QALYs). The various factors included in the model can be increased or decreased by a small amount one at a time. The one (possibly, but not likely, more than one) that improves the goal the most is the bottleneck(s) and any improvement initiative should be focused on it. The model can quantitatively predict the level of change in the goal for any change in the level of the bottleneck. However, it is not advisable to rush into elevating the bottleneck before exploiting the bottleneck and subordinating everything else to it, as per the steps two and three of TOC's process of ongoing improvement. Only at this point, if the constraint has not shifted, should it be elevated. And the process should restart to spot the new bottleneck.

Assume that it is determined that effective capacity is the bottleneck. Before elevating it (by adding more capacity), one might start by exploiting the bottleneck: Is the capacity run 
efficiently? If not, improving the utilization of the capacity could itself become the objective of a TOC's process of ongoing improvement. Several authors have reported enhancing effective capacity in the health industry by applying TOC's process of ongoing improvement including Kershaw (2000) and Womack and Flowers (1999). As part of this process of ongoing improvement, efficiency improvement initiatives including waste reduction techniques possibly through lean thinking (Womack and Jones, 2003) may be considered. Next, is everything else supporting the exploitation of the bottleneck? For instance, is the priority of health human resources providing health programs (as opposed to other activities such as teaching or conducting research)? Do the operating fund allocation policies support the exploitation of the bottleneck (e.g.: are the operating funds available to run the existing capacity all the time)? It is important to note here that this is where operating funds come into picture in our model, which is drastically different from how it was modeled by Schaefers et. al. (2007).

Continuing with the TOC's process of ongoing improvement, only when the subordination step has been completed and if the constraint has not shifted yet, should the effective capacity be enhanced (possibly through raising more capital funds and donations to increase physical capacity, or recruiting more health human resources). And then, the process should start from the beginning and the new bottleneck be identified by using the model.

Let's assume that the bottleneck has now shifted to health program effectiveness, meaning that improving the effectiveness of the health programs would improve health system performance the most. In exploiting the bottleneck, one might ask if health program effectiveness levels achieved in practice are in accordance with the latest effectiveness levels reported in the literature. If not, system wide initiatives to diffuse and enforce adoption of best practices should be put in place. If the level of health program effectiveness is low due to widespread medical 
errors, patient safety initiatives such as adopting computerized physician order entry to reduce medication errors might be considered. TOC's thinking process, as introduced by Goldratt (1994), has been helpful in identifying and addressing the root causes of medical errors as well (McNutt and Odwazny, 2004).

In the subordination step, other resources and initiatives should all prioritize the tasks aimed at improving the constraint over all other activities. For instance, health human resources capable of conducting medical research should prioritize medical research over other activities (e.g. teaching and providing health programs). Another example is to speed up the ethics approval process to expedite health research that can potentially increase the level of health program effectiveness. It is possible that with these three steps, the constraint has moved and the process should be started from step one: identifying the new constraint. If not, in step four of the process, more resources should be added to increase health program effectiveness, including more funding (or donation seeking) to conduct health or technological research that improve health program effectiveness.

\subsection{Discussion and Future Research}

There have been controversies around the concept and use of QALYs in the literature. Some interesting discussions can be found in Carr-Hill (1991), Carr-Hill and Morris (1991), Spiegelhalter et. al. (1992), Broome (1993), Nord (1993), Williams (1995), and more recently McGregor and Caro (2006). Some, such as McGregory and Caro (2006) question the QALYs methodology, mainly due to measurement difficulties, without proposing any other evidence based method. Others propose alternatives to QALYs, with each method having strengths and 
weaknesses associated with it. Mehrez and Gafni (1989, 1991, and 1992) discuss healthy-year equivalents (HYE). Saved-Young-Life equivalents (SAVE) are discussed by Nord et. al. (1993), and Nord (1995 , 1996). For the sake of our conceptual model, a unit of measurement is needed that captures both quality gains and quantity gains of life. While the choice of the right unit to measure quality and quantity of lives is in the realm of health economics and not the topic of this research, we chose to use QALY due its prevalence in the literature.

Once the unit of measurement is appropriately chosen, the model is indeed capable of addressing some of the most controversial issues in health policy. Take the public discussions around equity of access for instance. While in our conceptual model, we gave all members of society the same weight in aggregating the individual level models to construct the societal model, a similar idea to that of Williams (1995) by weighting individuals of the society differently (e.g. giving more weight to the poor or to the young) is also possible. We neither advocate nor object to different weightings of individuals in the society. All we say is that the model is capable of handling this policy issue, should a decision be made to weigh individuals differently.

The model is also capable of addressing the rationing issues. Should the effective capacity be less than that desired (as is the case for most health services in publicly funded health systems), the decision as to who should receive the health services should be made. While the conceptual model of TOC performance measures does not explicitly specify how to make these decisions, it includes the essential elements to address it. The TOC process of ongoing improvement would suggest exploiting and subordinating everything else to the effective capacity, as the constraint of the system, in times of rationing. Therefore, the TOC process of ongoing improvement would favor providing services to those most likely to benefit from them (i.e. those with higher "return on health investment” on every QALY invested in health services). If the amount of time wasted 
to receive health services is relatively the same for everybody, this could be equivalent to those with higher clinical priorities, as conceptualized with the "health program effectiveness" element of the model. We admit that it would take a bold politician or decision maker to implement such explicit rationing rules. But, on the positive side, it does bring the rationing discussion to the front and help the public make explicit decisions on how to maximize the goal. Of course, other considerations of the public, such as favoring sections of the population, can be made prior to moving to the societal model by giving different sub-populations different weights. In subordinating everything else to the constraint stage of the TOC, it would be advisable to offload the provision of health programs that could use less scarce resources to those resources, so that the scarcest capacity is saved for the ones that really need it. An example would be to offload the care of a diabetes patient who could benefit either from a specialist or a dietician to a dietician, when the supply of specialists is scarce.

We admit that while the model does provide the framework to address high level health policy issues, extensive research is still needed to create an accurate and complete model populated with evidence based estimates for the various model parameters from a wide variety of literature in health services research, such as clinical, health economics, workforce, health technology, and operational studies. However, one of the strengths of the model is that it does not necessarily need to be applied at the whole health system level. The conceptual model can be regarded as a framework and used at different levels: the high national or regional health system level, mesolevels for specific diseases (e.g. heart disease or cancer) or specific populations (e.g. seniors or particular ethnic groups), and the micro level of understanding individual patient decision making. It is possible that, within the current literature, there is sufficient evidence to populate the model for a specific level. 
Moreover, the model can be used as a qualitative tool as well. Indeed, influence diagrams used in system dynamics could have been used to present the model in the first place. Our choice of a stock and flow diagram was due to our desire to bring more clarity to the details of the performance measures. It is sometimes difficult to understand all the units of measurement in an influence diagram. Now that the level of clarity has been achieved, should decision makers see that there is not enough evidence to populate the model quantitatively at certain levels of its application; the model can be used as a qualitative tool to guide TOC's process of ongoing improvement. This is not the first time that a conceptual model without detailed formulations has been represented with stock and flow diagrams in the literature. For another example, please refer to Repenning and Sterman (2002).

A final discussion that we would like to present here is the potential applicability of our contributions to health systems that are not publicly funded. To the extent that the goal of such health systems is similar to that of the publicly funded health systems (e.g. some non-profit privately funded health systems), our conceptual framework of the system-level TOC performance measures would be useful for such health systems as well. However, if the goal of the health system is to maximize profit, then the classical TOC performance measures of cash flow, net profit, and return on investment would be applicable.

\subsection{Conclusions}

Theory of constraints has the potential to improve the resource-constrained publicly funded health systems by focusing improvement efforts on system constraints. However, to date, the literature has not adequately addressed the required customizations to make the theory applicable 
to this environment. Building upon a system dynamics representation of TOC's classical goal and performance measures for publicly traded for-profit companies, we developed a similar model that defined the goal and performance measures for publicly funded health systems. The model was then expanded, at a conceptual level, to include various factors affecting health system performance with the aim to guide the TOC's process of ongoing improvement. Future research should focus on developing more accurate models and populate them with parameters from a diverse set of health research literature to be able to actually use the model for guiding TOC’s process of ongoing improvement in health systems. 


\section{Connecting Low-Level Performance Measures to the Goal}

In chapter three we demonstrated how the drum-buffer-rope technique can balance the tradeoff between the two low-level performance measures of average patient wait time and instance of delayed treatment. However, the strategic question of what level of compromise in one low-level performance measure in favor of the other is acceptable remained unanswered. We stated that this question should be answered in the larger context of why these services are funded. In chapter four we defined the purpose of funding health services, and developed a set of systemwide performance measures to gauge the level of achieving the goal. In this chapter, we aim to bridge the gap in between the two chapters. Specifically, we would like to explore how linking the low-level performance measures to the system-wide measures can help answer the question of what level of compromise in one low-level performance measure in favor of the other is acceptable.

Figure 5.1 builds on figure 4.3 to demonstrate some of major effects of low-level performance measures on system-wide performance measures. Similar to our approach in chapter four, only the direction of the relationship between various variables is shown in figure 5.1. Recall that a positive sign on the arrow connecting $\mathrm{A}$ to $\mathrm{B}$ means that, all else equal, if $\mathrm{A}$ increases (decreases), then B “increases (decreases) above (below) what it would have been” (Sterman, 2000). A negative sign means that, all else equal, if A increases (decreases), then B “decreases (increases) below (above) what it would have been” (Sterman, 2000). 
The thick arrows on the left side of figure 5.1 are the new relationships added to figure 4.3. Essentially, the new relationships are as follows. The low-level performance measure of Average Patient Wait Time positively affects Total Quality Adjusted Wait Time, which itself positively affects Total QALYs Wasted. The low-level performance measure of Instances of Delayed Treatment positively affects Total Quality Adjusted Return Trip Time, which itself positively affects Total QALYs Wasted.

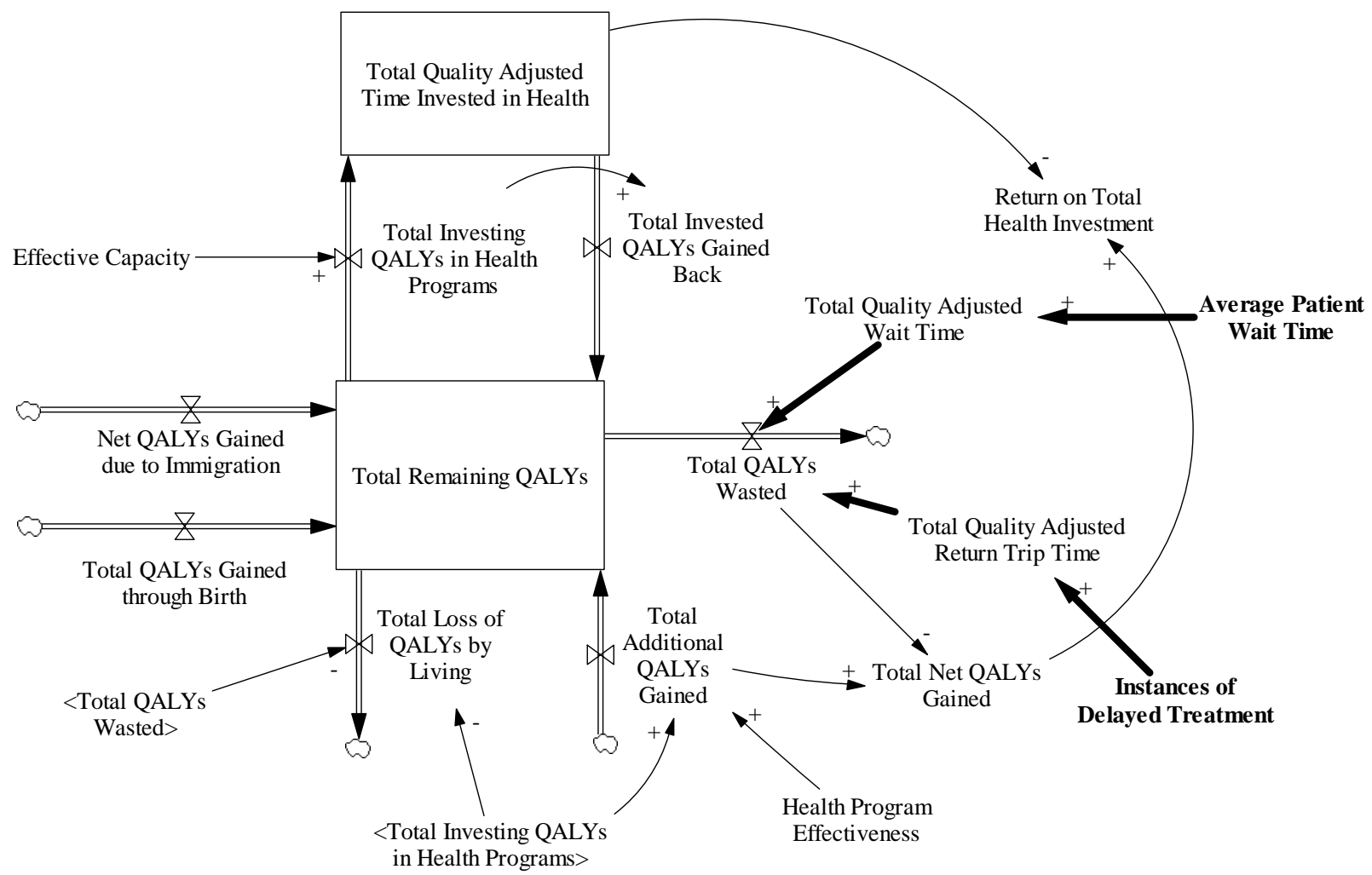

Figure 5.1: Low-Level \& System-Wide Performance Measures- Major Relationships

So what level of compromise in one low-level performance measure in favor of the other is acceptable? The right balance between the two low-level performance measures is the one that maximizes the goal. Since the new major relationships affect the goal through "Total QALYs 
Wasted”, the right balance between the two low-level performance measures is the one that minimizes the "Total QALYs Wasted". While finding this minimizing trade-off point theoretically might be difficult, in practice the discrete event simulation model presented in chapter three can be used to compare scenarios against each other.

For instance, to compare the scenarios that we discussed in chapter three, one might do a simple analysis with regards to their effects on the "Total QALYs Wasted" in the following manner: The two performance measures of average patient wait time and instances of delayed treatment can be combined to calculate total time wasted to receive treatment at the transfusion unit stage. Please note that this does not include the time wasted during the phases preceding the transfusion unit stage. However, since all the scenarios discussed in chapter three share the same wait times at other stages of the outpatient visit and only differ in the wait times and instances of delayed treatment at the transfusion unit stage, the scenario with the least amount of time wasted to receive the treatment at the transfusion unit stage is indeed the scenario that wastes the least amount of time (among the discussed scenarios) over the course of the day for all leukemia outpatients.

Taking the simulation results for the average patient wait time at the constrained stage for each of the scenarios, and assuming an average 1.5 hour return trip between the cancer facility and patients' residences, the total time wasted to receive treatment at the transfusion unit stage has been calculated and presented in table 5.1. It is seen that, among the listed scenarios, taking the $70^{\text {th }}$ percentile value of treatment chair availability times as the time estimator to schedule prebooked TFU patients wastes the least amount of patients' time overall. We conducted a sensitivity analysis on the 1.5 hour return trip time by changing it between an hour and two hours, and the $70^{\text {th }}$ percentile value remained the best among the scenarios. 


\begin{tabular}{|c|c|}
\hline $\begin{array}{c}\text { Percentile } \\
\text { Value }\end{array}$ & $\begin{array}{c}\text { Total Wasted Hours } \\
\text { to Receive Treatment at } \\
\text { the TFU }\end{array}$ \\
\hline 0 & 20.76 \\
\hline 10 & 19.37 \\
\hline 30 & 18.77 \\
\hline 50 & 18.43 \\
\hline 70 & 18.27 \\
\hline 90 & 18.46 \\
\hline 100 & 20.41 \\
\hline
\end{tabular}

\section{Table 5.1: Total Hours Wasted to Receive Treatment at the TFU in Different Scenarios}

In the above simple analysis, the time savings were not quality adjusted, whereas the systemwide measures are all quality adjusted. A more accurate analysis would have converted the time units (average patient wait time and average return trip time) to quality adjusted ones, and then combine the two low-level performance measures to create the total QALYs saved (i.e. less wasted) in one alternative compared to the other.

Moreover, in addition to the major relationships between the low-level performance measures and the system-wide ones discussed above, there are at least two more relationships that are possible. These are shown with the thick arrows in figure 5.2. 


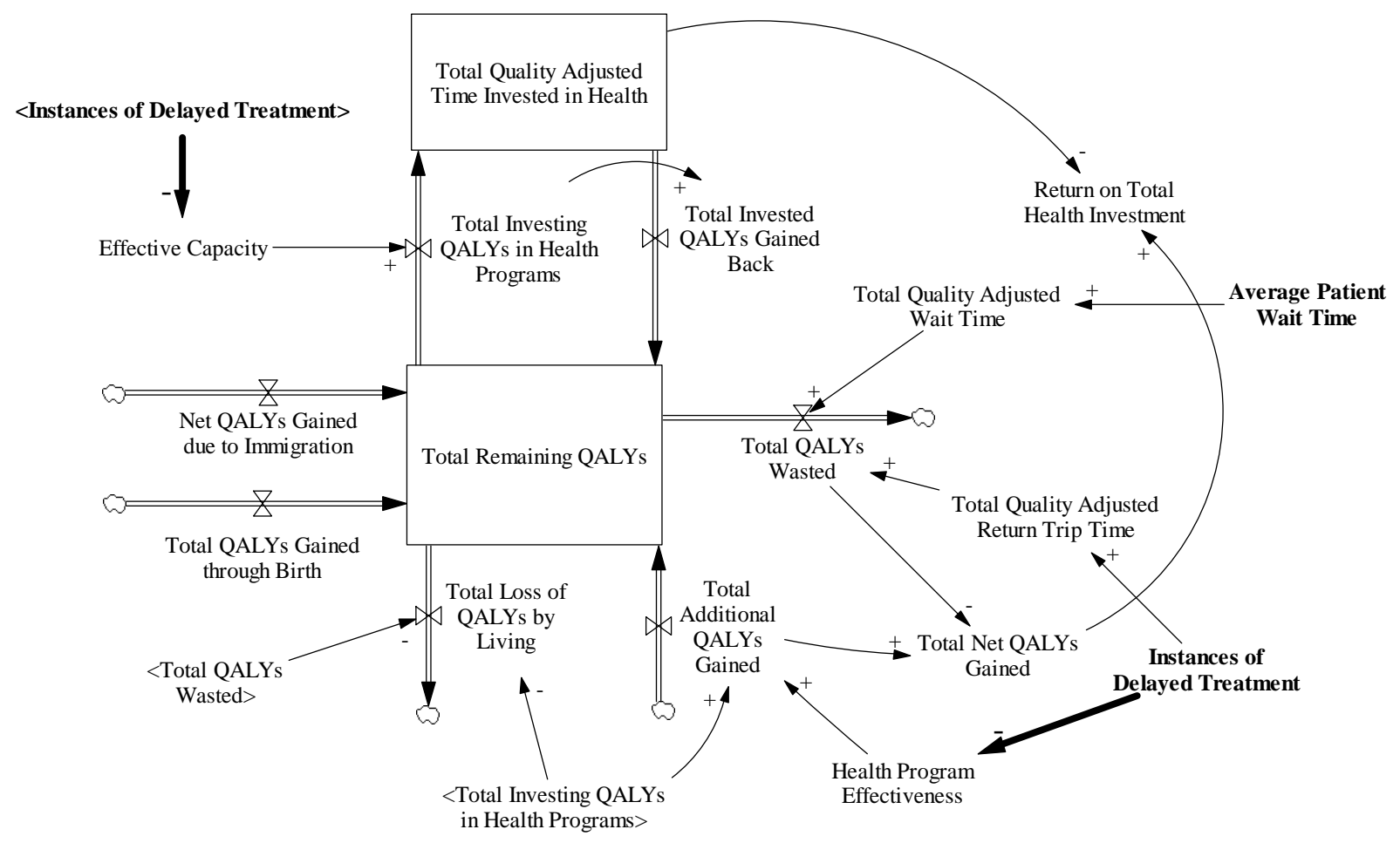

Figure 5.2: Low-Level \& System-Wide Performance Measures- Possible Relationships

On the right of figure 5.2, Instances of Delayed Treatment might negatively affect Health Program Effectiveness, meaning that a delayed treatment is not as effective as a same-day treatment. Since Health Program Effectiveness positively affects Total Additional QALYs Gained, the Instances of delayed Treatment would negatively affect Total Additional QALYs Gained.

This effect of Instances of Delayed Treatment on Health Program Effectiveness is only a possibility, since we could not find any studies in the literature affirming or rejecting this relationship for the case of delaying acute leukemia treatments. However, similar studies for other medical conditions exist. For instance, there is at least one study showing that delays in the start of radiotherapy treatment have an adverse effect on tumour control for fast-growing 
tumours (Wyatt et. al., 2008). So it is possible that delayed treatment in the case of acute leukemia patients could negatively affect Health Program Effectiveness as well.

More importantly, it is possible that an increase in the Instances of Delayed Treatment would negatively affect effective capacity, as depicted on the top left side of figure 5.2. The idea here is that if the Instances of Delayed Treatment are increased to the extent that the demand for next day services are far more than the capacity, then the effective capacity to treat patients the next day limits the start of the treatment process for some of next day's patients. So, Total Investing QALYs in Health Programs is reduced, which means that, assuming a positive Health Program Effectiveness, Total Additional QALYs Gained would be reduced or delayed in the future, thereby reducing the level of the goal. While due to both the structure of the data available and that of our discrete event simulation model we cannot quantify this effect, this is an important effect that should be quantified from the tax payers' perspective. As long as the instances of delayed treatment in any two scenarios being compared are about the same, it is reasonable to think that this effect does not materialize. But if a scenario changes the value of instances of delayed treatment to a great extent, and if the capacity is relatively tight compared to the demand, then this effect is a possibility.

We confess that estimating quality-adjusted time savings of the various scenarios, as well as quantifying the possible effects presented in figure 5.2, are not easy tasks and may require extensive multi-disciplinary research, possibly encompassing health economics, marketing, and medical expertise. However, we think linking low-level and system-wide measures is essential in transferring the theory of constraints to the setting of publicly funded health systems. After all, the promise of the theory is to enhance system performance through a set of tools and this cannot 
be accomplished without gauging the performance of each specific tool on the system-wide measures. 


\section{Conclusions}

This thesis aimed to fill the gap in the literature of the theory of constraints (TOC) in publicly funded health systems. In chapter two we identified two gaps in the literature with regards to the application of TOC to this setting: There are still no reported application of TOC's drum-bufferrope tool and inadequate customizations with regards to defining system-wide goal and performance measures.

The third chapter of the thesis, “Drum-Buffer-Rope for an Outpatient Cancer Facility”, presented a real world case study exploring the usefulness of TOC's drum-buffer-rope scheduling technique in a publicly funded outpatient cancer facility. With the use of a discrete event simulation model populated with historical data, the drum-buffer-rope scheduling policy was compared against "high constraint utilization" and "low wait time” scenarios. Drum-buffer-rope proved to be an effective mechanism in balancing the inherent tradeoff between the two performance measures of instances of delayed treatment and average patient wait time. To find the appropriate level of compromise in one performance measure in favor of the other, the linkage of these measures to system-wide performance measures, as developed in the fourth chapter of this thesis, were proposed.

The fourth chapter of the thesis, “Theory of Constraints’ Performance Measures for Publicly Funded Health Systems”, developed a system dynamics representation of the classical TOC's system-wide goal and performance measures for publicly traded for-profit companies, which formed the basis for developing a similar model for publicly funded health systems. The model 
was then expanded to include some of the factors that affect system performance, providing a framework to apply TOC's process of ongoing improvement in publicly funded health systems.

In chapter five of this thesis, we provided a framework to assess the effects of low-level performance measures on the global goal. Taking the specific example of our drum-buffer-rope technique in chapter three, we discussed what effects need to be quantified to connect the lowlevel performance measures to the system-wide measures. We admit that this linkage requires extensive further multidisciplinary research, possibly encompassing health economics, marketing, and medical expertise. However, we think that linking the low-level effects of various tools of the theory of constraints, such as the drum-buffer-rope technique, to the overall goal and performance measures is imperative in order to fully transfer the theory to the setting of publicly funded health system. After all, the promise of the theory is to enhance system performance through a set of tools and this cannot be accomplished without gauging the performance of each specific tool on the system-wide measures.

A final discussion that we would like to present here is the potential applicability of our contributions to health systems that are not publicly funded. To the extent that the goal of such health systems is similar to that of the publicly funded health systems (e.g. non-profit privately funded health systems), our conceptual framework of the system-level TOC performance measures presented in chapter four would be useful for such health systems as well. However, if the goal of the health system is to maximize profit, then the classical TOC performance measures of cash flow, net profit, and return on investment would be applicable.

With regards to the drum-buffer-rope tool discussed in chapter three, as long as the operating model of the organization is to balance the wait time and instances of delayed treatment, the DBR tool should be an appropriate one. Many private health organizations indeed might like to 
use their resources effectively, and at the same time provide a reasonable experience to patients. Such organizations would most likely benefit from the drum-buffer-rope technique. On the contrary, if the model of running the business is one of purely prioritizing one performance measure over the other (e.g. in case of some private health organizations, prioritizing patient experience over every other aspect of the business, in the hope to attract highly affluent patients to fund the system), then the drum-buffer-rope might be of limited use (unless extreme values of the percentiles are taken). In any case, the low-level performance measures of the operating model (e.g. average wait time and instances of delayed treatment) have to be linked to the system-wide measures of the specific system, which might be the classical TOC measures for some for-profit companies. 


\section{$7 \quad$ References}

Banks, J., J. S. Carson, B. L. Nelson, and D. M. Nicol. 2005. Discrete-event system simulation. 4th ed. ed. Upper Saddle River, NJ: Pearson Prentice Hall.

Belvedere, V., and Grando. 2005. Implementing a pull system in batch/mix process industry through theory of constraints: A case-study. Human Systems Management 24, (1): 3-12.

Blackstone, J. H., and J. F. Cox. 2002. Designing unbalanced lines-understanding protective capacity and protective inventory. Production Planning \& Control 13, (4): 416-23.

Bolander, S. F., and S. G. Taylor. 2000. Scheduling techniques: A comparison of logic. PRODUCTION AND INVENTORY MANAGEMENT JOURNAL 41, (1): 1-5.

Breen,A.M., Burton-Houle T., and Aron D.C. 2002. Applying the theory of constraints in health care: Part 1--the philosophy. Quality Management in.Health.Care. 10, (3): 40-6.

Broome, J. 1993. Qalys. Journal of Public Economics 50, (2): 149-76.

Carr-Hill, R. A., and J. Morris. 1991. Current practice in obtaining the" Q" in QALYs: A cautionary note. British Medical Journal 303, (6804): 699.

Carr-Hill, R. A. 1991. Allocating resources to health care: Is the QALY (quality adjusted life year) a technical solution to a political problem? International Journal of Health Services : Planning, Administration, Evaluation 21, (2): 351-63. 
Chakravorty, S. S. 2001. An evaluation of the DBR control mechanism in a job shop environment. Omega 29, (4): 335-42.

Dettmer, H. W. 1997. Goldratt's theory of constraints: A systems approach to continuous improvement American Society for Quality.

Drummond, M. F. 1987. Methods for the economic evaluation of health care programmes, eds. Greg L. Stoddart, George W. Torrance. New York: Oxford University Press.

Fung, KK. 1999. Follow the laggard? not all bottlenecks are created equal. System Dynamics Review 15, (4): 403-10.

Golden, B., H. Fraser, M. Leung, R. Hannam, and D. Soman. 2009. Designing your Patients' Experience: A Rotman Perspective. Work in Progress.

Goldratt, E. M. 1997. Critical chain. North River Press Great Barrington, MA.

Goldratt, E. M. 1994. It's not luck. North River Press Great Barrington, MA.

Goldratt, E. M. 1990. The haystack syndrome. North River Press Croton-on-Hudson, NY.

Goldratt, E. M., and J. Cox. 1984. The goal: Excellence in manufacturing. North River Press.

Goldratt, E. M., and R. E. Fox. 1986. The race. North River Press Croton-on-Hudson, NY.

Goldratt, R., and N. Weiss. 2005. Significant enhancement of academic achievement through application of the theory of constraints (TOC). Human Systems Management 24, (1): 13-9.

Guide, VDR. 1996. Scheduling using drum-buffer-rope in a remanufacturing environment. International Journal of Production Research 34, (4): 1081-91. 
Gun, Bruce, and S. Nahavandi. 2000. Determining the optimal level of work in progress using constraint analysis and computer simulation. Assembly Automation 20, (4): 305-12.

Gupta, M. , and Kaline. 2008. Managing a community mental health agency: A theory of constraints based framework. Total Quality Management \& Business Excellence 19, (3): 28194.

Gupta, M., L. Boyd, and L. Sussman. 2004. To better maps: A TOC primer for strategic planning. Business Horizons 47, (2): 15-26.

Gupta, M. 2003. Constraints management--recent advances and practices. International Journal of Production Research 41, (4): 647-59.

Hunink, M. G. 2001. In search of tools to aid logical thinking and communicating about medical decision making. Medical Decision Making 21, (4): 267.

Kalina-Kaminsky, C. 2005. A Statistical Analysis of the Theory of Constraints.

Kershaw, Russ. 2000. USING TOC TO "CURE" healthcare problems. Management Accounting Quarterly 1, (3) (Spring): 1-8.

Kim, S., V. J. Mabin, and J. Davies. 2008. The theory of constraints thinking processes: Retrospect and prospect. INTERNATIONAL JOURNAL OF OPERATIONS AND PRODUCTION MANAGEMENT 28, (2): 155.

Law, Averill M. 2007. Simulation modeling and analysis. 4th ed. ed. New York, NY: McGrawHill. 
Leshno, M., and B. Ronen. 2001. The complete kit Concept-Implementation in the health care system. Human Systems Management 20, (4): 313-8.

Louw, L., and D. C. Page. 2004. Queuing network analysis approach for estimating the sizes of the time buffers in theory of constraints-controlled production systems. International Journal of Production Research 42, (6): 1207-26.

Lubitsh, G., C. Doyle, and J. Valentine. 2005. The impact of theory of constraints (TOC) in an NHS trust. Journal of Management Development 24, (2): 116-31.

Mabin, V. J., and S. J. Balderstone. 2003. The performance of the theory of constraints methodology: Analysis and discussion of successful TOC applications. INTERNATIONAL JOURNAL OF OPERATIONS AND PRODUCTION MANAGEMENT 23, (5/6): 568-95.

Mabin, Victoria J., and S. J. Balderstone. 2000. The world of the theory of constraints : A review of the international literature. Boca Raton: St. Lucie Press.

McGregor, M., and J. J. Caro. 2006. QALYs: Are they helpful to decision makers? PharmacoEconomics 24, (10): 947.

McNutt, Robert A. MD, and Odwazny, Mary C. MS. 2004. The theory of constraints and medical error: A conversation with robert A. McNutt. Quality Management in Health Care 13, (3) (Jul-Sep): 183-7.

Mehrez, A., and A. Gafni. 1991. Healthy years equivalent: How to measure them using standard gamble approach. Medical Decision Making 11, : 140-6. 
Mehrez, A., and A. Gafni. 1990. Evaluating health related quality of life: An indifference curve interpretation for the time trade-off technique. Soc Sci Med 31, (11): 1281-3.

Mehrez, A., and A. Gafni. 1989. Quality adjusted life years (QALYs), utility theory, and healthy years equivalent (HYE). Med Decis Making 9, : 142-9.

Motwani, J., D. Klein, and R. Harowitz. 1996. Celebrate and record the theory of constraints in services: Part 2-examples from health care. Managing Service Quality 6, (2): 30-4.

Nord, E. 1996. Health status index models for use in resource allocation decisions. International Journal of Technology Assessment in Health Care 12, (1): 31-44.

Nord, E. 1995. The person-trade-off approach to valuing health care programs. Medical Decision Making 15, (3): 201.

Nord, E. 1993. Toward quality assurance in QALY calculations. International Journal of Technology Assessment in Health Care 9, (1): 37-45.

Nord, E., J. Richardson, and K. Macarounas-Kirchmann. 1992. Social evaluation of health care versus personal evaluation of health states: Evidence on the validity of four health state scaling instruments using norwegian and australian survey dataNational Centre for Health Program Evaluation.

Pass, S., and B. Ronen. 2003. Management by the market constraint in the hi-tech industry. International Journal of Production Research 41, (4): 713-24.

Patwardhan, M. B., A. Sarria-Santamera, and D. B. Matchar. 2006. Improving the process of developing technical reports for health care decision makers: Using the theory of constraints 
in the evidence-based practice centers. International Journal of Technology Assessment in Health Care 22, (1) (Winter): 26-32.

Radovilsky, Z. D. 1998. A quantitative approach to estimate the size of the time buffer in the theory of constraints. International Journal of Production Economics 55, (2): 113-9.

Rahman, S. 2002. The theory of constraints' thinking process approach to developing strategies in supply chains. Management 32, (10): 809-28.

Rahman, S. 1998. Theory of constraints: A review of the philosophy and its applications. International Journal of Operations \& Production Management 18, (4): 336-55.

Rand, G. K. 2000. Critical chain: The theory of constraints applied to project management. International Journal of Project Management 18, (3): 173-7.

Reid, R. A. 2007. Applying the TOC five-step focusing process in the service sector: A banking subsystem. Managing Service Quality 17, (2): 209-34.

Repenning, N. P., and J. D. Sterman. 2002. Capability traps and self-confirming attribution errors in the dynamics of process improvement. Administrative Science Quarterly: 265-95.

Ricketts, J. A. 2008. Reaching the goal: How managers improve a services business using goldratt's theory of constraints. IBM Press/Pearson.

Riggs, J. L. 1996. Engineering economics. McGraw-Hill New York.

Ronen, B., J. S. Pliskin, S. Pass, and I. NetLibrary. 2006. Focused operations management for health services organizations. Jossey-Bass. 
Rosolio, I., B. Ronen, and N. Geri. 2008. Value enhancement in a dynamic environment--a constraint management expert system for the oil refinery industry. International Journal of Production Research 46, (16): 4349-68.

Rotstein, Z., Wilf-Miron R., Lavi B., Seidman D.S., Shahaf P., Shahar A., Gabay U., and Noy S. 2002. Management by constraints: Considering patient volume when adding medical staff to the emergency department. The Israel Medical Association Journal : IMAJ 4, (3) (Mar): 1703.

Sale, M. L. Value Chain Analysis and Application of TOC to Service Organizations. Proceedings of the Academy of Production and Operations Management 2, (1) (2005).

Santibañez, P., V. Chow, J. French, M. Puterman, and S. Tyldesley. 2008. Reducing patient wait times \& improving resource utilization at the BC cancer Agency's ambulatory care unit through simulation.Working Paper

Schaefers, J., J. Colin, R. Aggoune, and M. Kucina. 2007. A contribution to performance measurement in the healthcare industry: The industrial point of view. International Journal of Business Performance Management 9, (2): 226-39.

Schmenner, R. W. 1986. How can service businesses survive and prosper? Sloan Management Review 27, (3) (Spring): 21-32.

Scoggin, J. M., R. J. Segelhorst, and R. A. Reid. 2003. Applying the TOC thinking process in manufacturing: A case study. International Journal of Production Research 41, (4): 767-97.

Shoemaker, T. E., and R. A. Reid. 2005. Applying the TOC thinking process: A case study in the government sector. Human Systems Management 24, (1): 21-37. 
Siha, Samia. 1999. A classified model for applying the theory of constraints to service organizations. Managing Service Quality 9, (4): 255-64.

Simatupang, T. M., A. C. Wright, and R. Sridharan. 2004. Applying the theory of constraints to supply chain collaboration. Supply Chain Management: An International Journal 9, (1): 5770.

Spiegelhalter, D. J., S. M. Gore, R. Fitzpatrick, A. E. Fletcher, D. R. Jones, and D. R. Cox. 1992. Quality of life measures in health care. III: Resource allocation. BMJ: British Medical Journal 305, (6863): 1205.

Srinivasan, M. M., W. D. Best, and S. Chandrasekaran. 2007. Warner robins air logistics center streamlines aircraft repair and overhaul. Interfaces 37, (1): 7.

Sterman, J. D. 2000. Business dynamics: Systems thinking and modeling for a complex world. Irwin/McGraw-Hill.

Taylor, ,L.J., and Churchwell L. 2004. Goldratt's thinking process applied to the budget constraints of a texas MHMR facility. Journal of Health.and Human Services Administration 26, (4): 416-37.

Taylor, L. J., B. J. Moersch, and G. M. Franklin. 2003. Applying the theory of constraints to a public safety hiring process. PUBLIC PERSONNEL MANAGEMENT 32, (3): 367-82.

Taylor, L. J., and D. Sheffield. 2002. Goldratt's thinking process applied to medical claims processing. Hospital Topics 80, (4): 13-21. 
Umble, M., E. Umble, and S. Murakami. 2006. Implementing theory of constraints in a traditional japanese manufacturing environment: The case of hitachi tool engineering. International Journal of Production Research 44, (10): 1863-80.

Umble, Michael, and ElisabethJ Umble. 2006. Utilizing buffer management to improve performance in a healthcare environment. European Journal of Operational Research 174, (2) (October 16, 2006): 1060-75.

Vijan, S. 2006. Should we abandon QALYs as a resource allocation tool? PharmacoEconomics 24, (10): 953.

Watson, K. J., J. H. Blackstone, and S. C. Gardiner. 2007. The evolution of a management philosophy: The theory of constraints. Journal of Operations Management 25, (2): 387-402.

Williams, A. 1995. Economics, QALYs and medical ethics-A health economist's perspective. Health Care Analysis 3, (3): 221-6.

Womack, J. P., and D. T. Jones. 2003. Lean thinking: Banish waste and create wealth in your corporation. Free Press.

Womack, ,D.E., and Flowers S. 1999. Improving system performance: A case study in the application of the theory of constraints. Journal of Healthcare Management / American College of Healthcare Executives 44, (5) (Sep-Oct): 397,405; discussion 405-7.

Wright, J., and R. King. 2006. We all fall down. North River Press.

Wyatt, R. M., B. J. Jones, and R. G. Dale. 2008. Radiotherapy treatment delays and their influence on tumour control achieved by various fractionation schedules. British Journal of Radiology 81, (967): 549. 
Yang, J. B. 2007. How the critical chain scheduling method is working for construction. Cost Engineering(Morgantown, W.Va.) 49, (4): 25-32.

Ye, T. 2007. Determination of buffer sizes for drum-buffer-rope (DBR)-controlled production systems. International Journal of Production Research 46, (10): 2827-44.

Young,T., Brailsford S., Connell C., Davies R., Harper P., and Klein J.H. 2004. Using industrial processes to improve patient care. BMJ (Clinical Research Ed.) 328, (7432) (Jan 17): 162-4. 